\title{
QUALITY OF COMMERCIAL PALM-BASED COOKING OIL PACKED IN PLASTIC POUCH AND POLYETHYLENE TEREPHTHALATE (PET) BOTTLE
}

\author{
NORAZURA AILA MOHD HASSIM*; NUR HAQIM ISMAIL*; SIVARUBY KANAGARATNAM*; \\ WAN ROSNANI AWG ISA* and NOOR LIDA HABI MAT DIAN*
}

\begin{abstract}
The study compared the quality of palm cooking oil sold in two types of packaging in Malaysia; plastic pouch (16 samples) and polyethylene terephthalate (PET) bottle (9 samples). The study was conducted because consumer perceived that the quality of palm cooking oil in plastic pouch is lower than in the PET bottle due to its lower price. Cooking oil in plastic pouch is subsidised by the Government of Malaysia and as such, it is sold at a cheaper price compared to bottled cooking oil. Therefore, comparison of the initial quality in terms of free fatty acid (FFA), fatty acid composition (FAC), triacylglycerol composition (TAG), total vitamin E, iodine value (IV), cloud point, smoke point, colour, polar compound, polymer compound and oxidative stability index (OSI) of the commercial palm cooking oils were conducted between the cooking oil in plastic pouch and PET bottle. The quality parameters of average FFA, FAC (oleic, linoleic, palmitic and stearic acids), IV and colour (red and yellow) of cooking oil in both packagings met the specifications or guidelines by either Malaysian Standard (MS) 682:2004, MS 816:2007 or Palm Oil Refiners' Association (PORAM) specifications/guidelines. However, quality parameters of TAG, total vitamin $E$, cloud point, smoke point, polar compound, polymer compound and OSI are not specified in any of the standards above. After conducting a 2-sample $t$-test to detect differences of cooking oil in both packagings, quality parameters of average FFA, FAC (oleic acid), total vitamin E, colour (red, yellow, neutral and blue), polar compound and polymer compound were comparable between cooking oil in plastic pouch and PET bottle. However, the FAC (palmitic acid, stearic acid and linoleic acid), TAG [UUU (unsaturated-unsaturated-unsaturated) and SUU (saturated-unsaturated-unsaturated)], IV and cloud point quality of cooking oil in PET bottle were better than in plastic pouch in which FAC (stearic acid and linoleic acid), TAG [UUU (unsaturated-unsaturated-unsaturated) and SUU (saturated-unsaturated-unsaturated)] and IV in PET bottle showed higher value while and FAC (palmitic acid) and cloud point showed lower value than plastic pouch in this study. Notwithstanding this, the smoke point and OSI quality of commercial palm cooking oil in plastic pouch was better than in PET bottle, having higher values than plastic pouch. TAG [SUS (saturated-unsaturated-saturated)] of commercial cooking oil in plastic pouch was also higher than in PET bottle but did not necessarily provide indication of good quality cooking oil.
\end{abstract}

Keywords: palm cooking oil, palm olein, palm superolein, packed in plastic pouch, packed in PET bottle.

Received: 10 July 2020; Accepted: 21 August 2020; Published online: 15 October 2020

\section{INTRODUCTION}

There are 17 oils and fats produced and traded globally. Palm oil is regarded as one of the most

\footnotetext{
Malaysian Palm Oil Board,

6 Persiaran Institusi, Bandar Baru Bangi,

43000 Kajang, Selangor, Malaysia.

E-mail: azuraaila@mpob.gov.my
}

important commodities, contributing to $57 \%$ of the global exportation of oils and fats in 2019 (MPOB, 2020). Palm oil and its fractions are vastly used in the food segment. Refined, bleached and deodourised (RBD) palm olein with iodine value (IV) of 56-59, the liquid fraction of palm oil from the first stage fractionation, has been widely used as cooking/ frying oil especially in Malaysia due to its availability, 
thermal stability and price competitiveness. Many studies have been conducted to look at the frying performance of palm olein during batch and continuous frying of food. Studies by Ahmad Tarmizi and Ismail (2008); Matthäus (2007); Ismail (2005); Sebedio et al. (1996) and Bracco et al. (1981) have shown that palm olein is a good and durable cooking oil. However, the number of studies conducted on frying performance of palm olein in batch frying had superseded continuous frying, possibly due to the costly operation of the latter. Palm olein is generally viewed as a heavy duty frying oil, which is commonly re-used in the snack food industry (Ahmad Tarmizi and Ismail, 2008; Nallusamy, 2006). Notwithstanding this, super olein with IV of $60-67$, which is a liquid fraction of palm oil from the second stage fractionation produced by certain refineries, is also part of the cooking oil source.

Cooking oil functions as a medium of heat transfer to the food surface, with some of it being absorbed by the food thus ensuring its quality becomes very important. Despite that some of the oil is absorbed by the food (amount of absorption depends on the type of matrix and frying practices), household and industry are at great extend still practice cooking using oil as the process generates pleasing sensory properties of the food. As degradation (i.e. oxidation, hydrolysis, polymerisation, dehydration and cyclisation) occurs during the frying process, the initial quality of the oil has to be ensured before the cooking process. In this regards, Malaysian Standard (MS) 682:2004 (Department of Standards Malaysia, 2004) specifies requirements for cooking oil, covering blended and unblended cooking oil (refined and deodourised) which comprise free fatty acids (FFA), volatile matter and impurities, soap content, unsaponifiable matter and colour. Notwithstanding this (MS) 816:2007 (Department of Standards Malaysia, 2007) specifies the requirement for palm olein, covering parameters of apparent density, refractive index, saponification value, unsaponifiable matter, fatty acid composition (FAC), IV, slip melting point (SMP), total carotenoids, FFA, moisture and impurities, peroxide value and colour (Lovibond).

In Malaysia, there are two types of packaging for cooking oil, namely the plastic pouch/ packet and polyethylene terephthalate (PET) bottle. The price of cooking oil in plastic pouch/ packet packaging is subsidised and controlled by the Government of Malaysia and therefore, is cheaper than the price of PET bottled cooking oil. However, due to this scenario, consumers and consumer associations in Malaysia often regard cooking oil in pouch packaging as having lower quality compared to cooking oil in PET bottle. In view of this scenario and to correct the public misperception, several experiments have been conducted to analyse, confirm and compare the quality of cooking oil in both forms of packaging. Some crucial characteristics of palmbased cooking oil as mentioned in the Malaysian Standards, namely FFA, FAC, IV and colour and some additional crucial quality characteristics namely cloud point, smoke point, polar compound, polymer compound, oxidative stability index (OSI) and vitamin $\mathrm{E}$ (tocotrienols and tocopherols) in both plastic pouch and PET bottle were reported. Triacylglycerols (TAG) composition of the cooking oils was also analysed.

\section{MATERIALS AND METHODS}

\section{Materials}

The cooking oils (16 plastic pouches and nine $1 \mathrm{~kg}$ PET bottles) were purchased from Malaysian retail shops and hypermarkets. All chemicals used were analytical grade except for TAG analysis which used HPLC grade solvent.

\section{Free Fatty Acid}

The FFA value was determined according to the American Oil Chemists Society (AOCS) Official Method Ca 5a-40 (Firestone, 1998) using titration method. Oil sample $(20 \mathrm{~g})$ was dissolved in $50 \mathrm{ml}$ isopropanol and $2 \mathrm{ml}$ phenolphthalein indicator solution. $0.1 \mathrm{M}$ sodium hydroxide is titrated to the mixture until first permanent colour of pink appeared. This colour must remain for at least $30 \mathrm{~s}$. The FFA was calculated based on the percentage of palmitic acid.

\section{Fatty Acid Composition}

The FAC was determined using Gas Chromatography according to ISO 5508:1990 (1990) as fatty acid methyl ester (FAME) by dissolving 0.1 g samples with $1.8 \mathrm{ml}$ of hexane before methylated it with sodium methoxide. The process followed with the addition of water. Mixing process using vortex mixer was performed intermittently between each step for $10 \mathrm{~s}$. One $\mu \mathrm{l}$ FAME from sedimentation of the sodium glycerolate was injected into DuraBond DB-23 fused silica capillary column $(60 \mathrm{~m} \times 0.25$ $\mathrm{mm} \times 0.25 \mu \mathrm{m}$ ) (Agilent Technologies, Model 7890B) that was equipped with flame ionisation detector (FID). The detection temperature was set at $240^{\circ} \mathrm{C}$ while the oven temperature was set isothermally at $180^{\circ} \mathrm{C}$. The flow rate of helium gas was at $0.8 \mathrm{ml} \mathrm{min}{ }^{-1}$. FAME standards were used for peak identification followed with the peak area normalisation method of the quantified area. 


\section{Triacylglycerols Composition}

The TAG composition was determined using ultra high-performance liquid chromatography (U-HPLC) system (1290 Infinity LC System, Agilent Technologies, USA) and Agilent 1260 RI detector (Waters Corp., USA) according to AOCS Official Method Ce 5b-89 (Firestone, 1998). A Cortecs UPLC C18 column (2.1 mm x 150 mm length i.d; $1.6 \mu \mathrm{m}$ particle size) (Waters Corp., Milford, Massachusetts, USA) maintained at $30^{\circ} \mathrm{C}$ was used to separate the TAG. The isocratic mobile phase used was a mixture of acetone-acetonitrile at 63.5:36.5 (v/v) ratio at a flow rate of $0.25 \mathrm{ml} \mathrm{min}^{-1}$. Samples $(0.1 \mathrm{~g})$ was solubilised in $1 \mathrm{ml}$ of acetone. Identification of individual peak was carried out by comparing the retention times of pure TAG standards and typical palm olein composition. Data was recorded as percent areas.

\section{Vitamin E}

Total vitamin E, tocotrienols and tocopherols were determined according to AOCS Official Method Ce 8-89 (Firestone, 1998) using high performance liquid chromatography (HPLC) (Gilson) fitted with a $250 \mathrm{~mm} \times 4.6 \mathrm{~mm}$ column, packed with 5 $\mu \mathrm{m}$ of silica (Phenomenex, Torrance, USA). The sample was dissolved in $n$-hexane prior injecting into HPLC. Ratio of $n$-hexane and isopropanol at 99.6:0.04 (Merck, Germany) was used as mobile

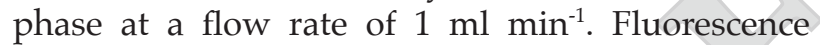
detector was used to detect vitamin $\mathrm{E}$ at an excitation and emission wavelengths of $290 \mathrm{~nm}$ and $330 \mathrm{~nm}$ respectively. Comparison of the retention time of Standard Tocopherols and Tocotrienols was used to identify the tocols (Isomer Kits of ChromaDex, Santa Ana, USA).

\section{Iodine Value (by calculation)}

IV was calculated according to the FAC factors as described by Ham et al. (1998) using the formulation as below:

$\mathrm{IV}=\left(0.95^{*} 16: 1\right)+\left(0.86^{*} 18: 1\right)+(1.72 * 18: 2)+(2.6 * 18: 3)$ Note: * is multiply; $16: 1,18: 1,18: 2$ and $18: 3$ is the value of each fatty acid.

\section{Cloud Point}

MPOB Test Method was used to determine the cloud point (MPOB, 2005). Oil sample of 60-75 g was filtered using Whatman No.1 filter paper and heated for $5 \mathrm{~min}$ at $130^{\circ} \mathrm{C}$ for foreign matter and moisture removal. The sample $(45 \mathrm{ml})$ was then put in a Beatson bottle, cooled (using water bath) and stirred until it reached $10^{\circ} \mathrm{C}$. More vigorous stirring was applied below $10^{\circ} \mathrm{C}$ when near to the expected cloud point, to avoid supercooling and fat crystals solidification on the bottle surface (side and bottom). The sample was taken out from the water bath at certain intervals to check for the sample cloudiness. The test was repeated at $5^{\circ} \mathrm{C}$ (below recorded cloud point). Repeated test runs by a single operator should not vary by more than $0.6^{\circ} \mathrm{C}$. The reading of the cloud point was taken once the thermometer immersed in the oil was not visible anymore.

\section{Smoke Point}

The smoke point was determined based on AOCS Official Method Cc 9a-48 (Firestone, 1998) using the Cleveland open-cup method. The oil sample was poured into the cup (usually brass) until the filling line and light beam was arranged at the centre of the cup. Thermometer is positioned at the centre of the cup and the cup was rapidly heated to $40^{\circ} \mathrm{C}-50^{\circ} \mathrm{C}$. This is followed by regulating the heating at a rate of $5^{\circ} \mathrm{C} \mathrm{min}^{-1}$. The increased temperature will cause the oil to produce smoke. The temperature at which the sample produces smoke is recorded as the smoke point.

\section{Colour}

The colour of oil samples was measured using Lovibond Tintometer Model F (The Tintometer Ltd., Salisbury, United Kingdom) according to AOCS Official Method Cc 13e-92 (Firestone, 1998). Glass cells, $51 / 4$-in. and 1-in. (13.3 and $2.5 \mathrm{~cm}$ ) path lengths, were used for containing the test samples. Cooking oil samples were heated in an oven at $60 \pm 2^{\circ} \mathrm{C}$ for $1 \mathrm{hr}$. The liquid oil was poured into a glass cell. The cell containing the sample was placed within the lighting cabinet. The lid of the lighting cabinet was closed and colour of the cooking oil sample was determined immediately using colour racks at a ratio of 10 yellow $(Y)$ to 1 red $(R)$ as the base line.

\section{Polar Compound}

Polar compounds were determined according to the Standard IUPAC Method 2.507 (IUPAC, 1987) using silica column chromatography gravimetrically. A $2.5 \mathrm{~g}$ oil sample was dissolved in $50 \mathrm{ml}$ elution solvent having $87: 13 \mathrm{v} / \mathrm{v}$ ratio of petroleum benzene and diethyl ether respectively. A $20 \mathrm{ml}$ of the oil sample solution prepared above was introduced onto a 4.5-m glass column filled with a slurry of Silica Gel 60 (Merck, Darmstadt, Germany), sea sand and the elution solvent. Elution of non-polar fraction is carried out with $150 \mathrm{ml}$ of the elution solvent within 60-70 min. The non-polar mixture was then collected in a flask. Removal of the solvent from the non-polar mixture was carried out using rotary evaporator. Nitrogen was used to cool the residue 
at room temperature by flushing it in the flask. The determination of polar compounds was conducted by subtracting the non-polar compounds from the mass of the sample dissolved in the petroleum ether and diethyl ether mixture.

\section{Polymer Compound}

Polymer compounds were quantified according to Peled et al. (1975). The oil sample was added with $1 \%$ sulphuric acid (Systerm, Shah Alam, Malaysia) before refluxed for $2.5 \mathrm{hr}$. The methanolic layer was separated from the semi-solid layer after cooling for $2.5 \mathrm{hr}$. The semi solid layer was then rinsed using methanol (Systerm, Shah Alam, Malaysia) and mixed with chloroform (LAB-SCAN, Dublin, Ireland) before being transferred to the conical flask. Chloroform was evaporated using a rotary evaporator and the remaining residue was dried at $130^{\circ} \mathrm{C}$ for $30 \mathrm{~min}$. The dried residue was weighed after being cooled at room temperature.

\section{Oxidative Stability Index}

Induction period was determined using a 743 Rancimat (Metrohm, Herisau, Switzerland) according to AOCS Official Method Cd 12b-92 (Firestone, 1998). Sample was weighed about 0.3 $\mathrm{g}$ and put at the bottom of the reaction vessel and heated at $110^{\circ} \mathrm{C}$ with a gas flow of 20 litre $\mathrm{hr}^{-1}$. The volatile products which were released during the oxidation process were collected in the flask containing $60 \mathrm{ml}$ of distilled water. A chart of water conductivity us. time was expressed from a multichannel strip chart recorder and the induction period was affirmed by a micro-processor computed slope algorithm. The induction period is prescribed as the point for which rapid transition as a result of oxidation, and expressed in hour (hr) (Aladedunye et al., 2014).

\section{Statistical Analysis}

FFA, TAG composition, smoke point, colour, polar compound, polymer compound and oxidative stability index were carried out in duplicate while FAC, vitamin E, IV and cloud point were carried out in triplicate. Results were analysed with Minitab version 16.0 (Pennsylvania, USA) using oneway analyses of variance (ANOVA). Significant differences $(\mathrm{p} \leq 0.05)$ among all samples (total $=25)$ were analysed using the Tukey's Post-Hoc test. Results were also analysed using 2-sample t-test with similar Minitab version. Significant differences $(\mathrm{p} \leq 0.05)$ between two groups (i.e. plastic pouch and PET bottle) were analysed. Pearson correlation was used to assess linear correlation between two continuous variables and was used to obtain correlation between certain parameters only.

\section{RESULTS AND DISCUSSIONS}

\section{Free Fatty Acid}

One of the quality parameters of cooking oil is FFA. Low FFA is preferable as high FFA produces off-flavour which makes the oil less acceptable. FFA is mainly produced through hydrolysis of TAG. However, some FFA may come from decomposition of hydroperoxide of the cooking oil at elevated temperature together with the presence of air and moisture (Bensmira et al., 2007). The FFA of commercial cooking oils in both plastic pouch and PET bottle is shown in Table 1a. Cooking oils in both plastic pouch and PET bottle have FFA values which ranged from $0.064 \%-0.205 \%$ and $0.77 \%-0.227 \%$, respectively. This makes the overall range of cooking oil in both packaging to be between $0.064 \%-0.227 \%$ and was significantly different $(\mathrm{p}<0.05)$ based on ANOVA. However, there were cooking oils that were not significantly different from each other. Sample V (PET bottle) has the highest FFA followed by sample A (plastic pouch) and B (plastic pouch). Sample K (plastic pouch) has the lowest FFA value. Malaysia Standard for Palm Olein - MS 816:2007 (Department of Standards Malaysia, 2007) and Palm Oil Refiners Association of Malaysia (PORAM) has put a limit of $0.1 \%$ FFA for RBD palm olein. Malaysian Standard for Cooking Oils - MS 682:2004 (Department of Standards Malaysia, 2004) has also set a maximum limit of unblended cooking oil of $0.2 \%$ while for blended cooking oil is $1.0 \%$. There was also recommendation by Gunstone (2008) that refined oils should have FFA value of less than $0.1 \%$. This is due to refining process that FFA is removed from crude oils. Samples that met PORAM's and Gunstone (2008) criteria were samples H, J, K, M and $\mathrm{N}$ (plastic pouch) and sample S and Y (PET bottle). Higher FFA limits $(0.2 \%)$ set by the Malaysian Standard for Cooking Oil-MS 682:2004(Department of Standards Malaysia, 2004) for unblended cooking oil was the most fulfilled standard by the cooking oil in this study. It was fulfilled by samples C, D, E, F, G, I, L, O, P (plastic pouch) and samples Q, R, T, $\mathrm{U}$ and $\mathrm{W}$ (PET bottle). Samples V and X (PET bottle) which were blended cooking oils had fulfilled the Malaysian Standard for Cooking Oil - MS 682:2004 (Department of Standards Malaysia, 2004) for blended cooking oil having limit of 1.0\% FFA. There were two samples (sample A and B in plastic pouch) that were unable to fulfill any standard or reference. Sometimes, the FFA value of commercial RBD palm olein may not fulfill any standards or references as the cooking oil has been on the shelf for a long time. An example of this was the cooking oil from the supermarket in Konya, Turkey used by Arslan et al. (2017) which recorded the FFA value of $0.77 \%$ (higher than Malaysian Standard and PORAM). Notwithstanding this, researches that used RBD 
palm olein direct from refineries normally obtained FFA value of less than $0.1 \%$ (i.e. Roiaini et al, 2015). A 2-sample t-test (Table $1 b$ ) showed that the average FFA of cooking oils in both plastic pouch and PET bottle was not significantly different $(p>0.05)$ and therefore, was comparable.

\section{TABLE 1A. FREE FATTY ACID (FFA) OF COMMERCIAL PALM-BASED COOKING OIL IN PLASTIC POUCH AND POLYETHYLENE TEREPHTHALATE (PET) BOTTLE PACKAGING IN MALAYSIA}

\begin{tabular}{cc}
\hline Sample & Free fatty acid $(\%)$ \\
\hline A (PP) & $0.208 \pm 0.004^{\mathrm{b}}$ \\
B (PP) & $0.205 \pm 0.000^{\mathrm{b}}$ \\
C (PP) & $0.141 \pm 0.000^{\mathrm{fg}}$ \\
D (PP) & $0.102 \pm 0.000^{\mathrm{i}}$ \\
E (PP) & $0.131 \pm 0.005^{\mathrm{g}}$ \\
F (PP) & $0.192 \pm 0.000^{\mathrm{c}}$ \\
G (PP) & $0.179 \pm 0.000^{\mathrm{d}}$ \\
H (PP) & $0.099 \pm 0.005^{\mathrm{i}}$ \\
I (PP) & $0.115 \pm 0.000^{\mathrm{h}}$ \\
J (PP) & $0.927 \pm 0.005^{\mathrm{i}}$ \\
K (PP) & $0.064 \pm 0.000^{\mathrm{k}}$ \\
L (PP) & $0.192 \pm 0.000^{\mathrm{c}}$ \\
M (PP) & $0.093 \pm 0.005^{\mathrm{i}}$ \\
N (PP) & $0.077 \pm 0.000^{\mathrm{j}}$ \\
O (PP) & $0.166 \pm 0.000^{\mathrm{e}}$ \\
P (PP) & $0.157 \pm 0.004^{\mathrm{e}}$ \\
Q (PB) & $0.166 \pm 0.000^{\mathrm{e}}$ \\
R (PB) & $0.141 \pm 0.000^{\mathrm{fg}}$ \\
S (PB) & $0.077 \pm 0.000^{\mathrm{j}}$ \\
T (PB) & $0.191 \pm 0.000^{\mathrm{c}}$ \\
U (PB) & $0.187 \pm 0.007^{\mathrm{cd}}$ \\
V (PB) & $0.227 \pm 0.004^{\mathrm{a}}$ \\
W (PB) & $0.191 \pm 0.000^{\mathrm{c}}$ \\
X (PB) & \\
Y (PB) & \\
\hline & $0.144 \pm 0.004^{\mathrm{f}}$ \\
\hline
\end{tabular}

Note: Sample A-P - cooking oils in plastic pouch (PP). Sample Q-Y - cooking oils in polyethylene terephthalate (PET) bottle (PB).

Means within each row bearing different superscript letters are significantly different $(\mathrm{p}<0.05)$.

TABLE 1B. 2-SAMPLE T-TEST OF FREE FATTY ACID (FFA) OF COMMERCIAL PALM-BASED COOKING OIL IN PLASTIC POUCH AND POLYETHYLENE TEREPHTHALATE (PET) BOTTLE PACKAGING IN MALAYSIA

\begin{tabular}{ccc}
\hline \multicolumn{2}{c}{ Mean of FFA $(\%)$} & p-value \\
\cline { 1 - 2 } Plastic pouch & PET bottle & \\
\hline $0.138 \pm 0.047$ & $0.156 \pm 0.051$ & 0.238 \\
\hline
\end{tabular}

Note: $p$-value $<0.05$ indicates that there is significant different between cooking oil in plastic pouch and PET bottle.

\section{Fatty Acid Composition}

Monitoring of FAC is usually conducted to confirm the authenticity of an oil or fat, as well as to know the stability and physicochemical properties of these products (Kostik et al., 2013). In this survey, confirmation on palm cooking oil whether single or blended oil(s) and their fraction(s) can be determined using FAC. The FAC of all cooking oils (in plastic pouch and PET bottle) is shown in Table $2 a$. Oleic acid was the major FAC of cooking oil in both plastic pouch and PET bottle, having value of between $41.68 \%-45.52 \%$ and $42.28 \%-45.29 \%$, respectively. This was followed by palmitic acid (between $34.79 \%-41.12 \%$ for plastic pouch and $35.64 \%-38.76 \%$ for PET bottle) and linoleic acid (between $10.66 \%-13.37 \%$ for plastic pouch and $12.07 \%-14.22 \%$ for PET bottle). FAC of all cooking oils (plastic pouch and PET bottle) was significantly different $(p<0.05)$ based on ANOVA. However, there were cooking oils that were not significantly different from each other. These fatty acid trends were similar to the finding by Ahmad Tarmizi et al. (2014) on palm olein. Samples H (plastic pouch), L (plastic pouch), V (PET bottle) and O (plastic pouch) contained the highest level of oleic acid, palmitic acid, linoleic acid and stearic acid respectively. Samples O (plastic pouch), H (plastic pouch), M (plastic pouch) and F (plastic pouch) on the other hand, had the lowest level of oleic acid, palmitic acid, linoleic acid and stearic acid accordingly. Lauric, myristic, palmitoleic, linolenic and arachidic acid was found present but in very small quantity. FAC of cooking oil in both pouch and PET bottle should resemble the value of palm olein or palm superolein as both fractions are the common raw materials for cooking oil.

According to Malaysian Standard for Palm Olein - MS 816:2007 (Department of Standards Malaysia, 2007), RBD palm olein should have oleic acid value of $39.8 \%-43.9 \%$, palmitic acid of $38.2 \%-$ $42.9 \%$, linoleic acid of $10.4 \%-12.7 \%$ and stearic acid of $3.7 \%-4.8 \%$ in which majority of the dominant FAC in the commercial cooking oil (plastic pouch and PET bottle) fall in this range. However, comparison to this standard indicated that samples $\mathrm{H}, \mathrm{J}$ and $\mathrm{P}$ (plastic pouch) and Q, R, S, T, U, V, W and Y (PET bottle) have lower value of palmitic acid, samples C, D, H and P (plastic pouch) and R and W (PET bottle) have higher value of oleic, samples $\mathrm{H}$ (plastic pouch) and $S, U$ and $\mathrm{V}$ (PET bottle) have higher range of linoleic acid and samples $\mathrm{D}, \mathrm{F}, \mathrm{H}$ and $\mathrm{P}$ (plastic pouch) have lower range of stearic acid. Notwithstanding this, based on 2-sample t-test, the average palmitic acid, stearic acid and linolenic acid of cooking oil in both packagings were significantly different $(\mathrm{p}<0.05)$ (Table $2 b)$. However, the average of oleic acid was comparable for cooking oil in both packaging ( $\mathrm{p}>0.05)$. Tabee et al. (2009) stated that 


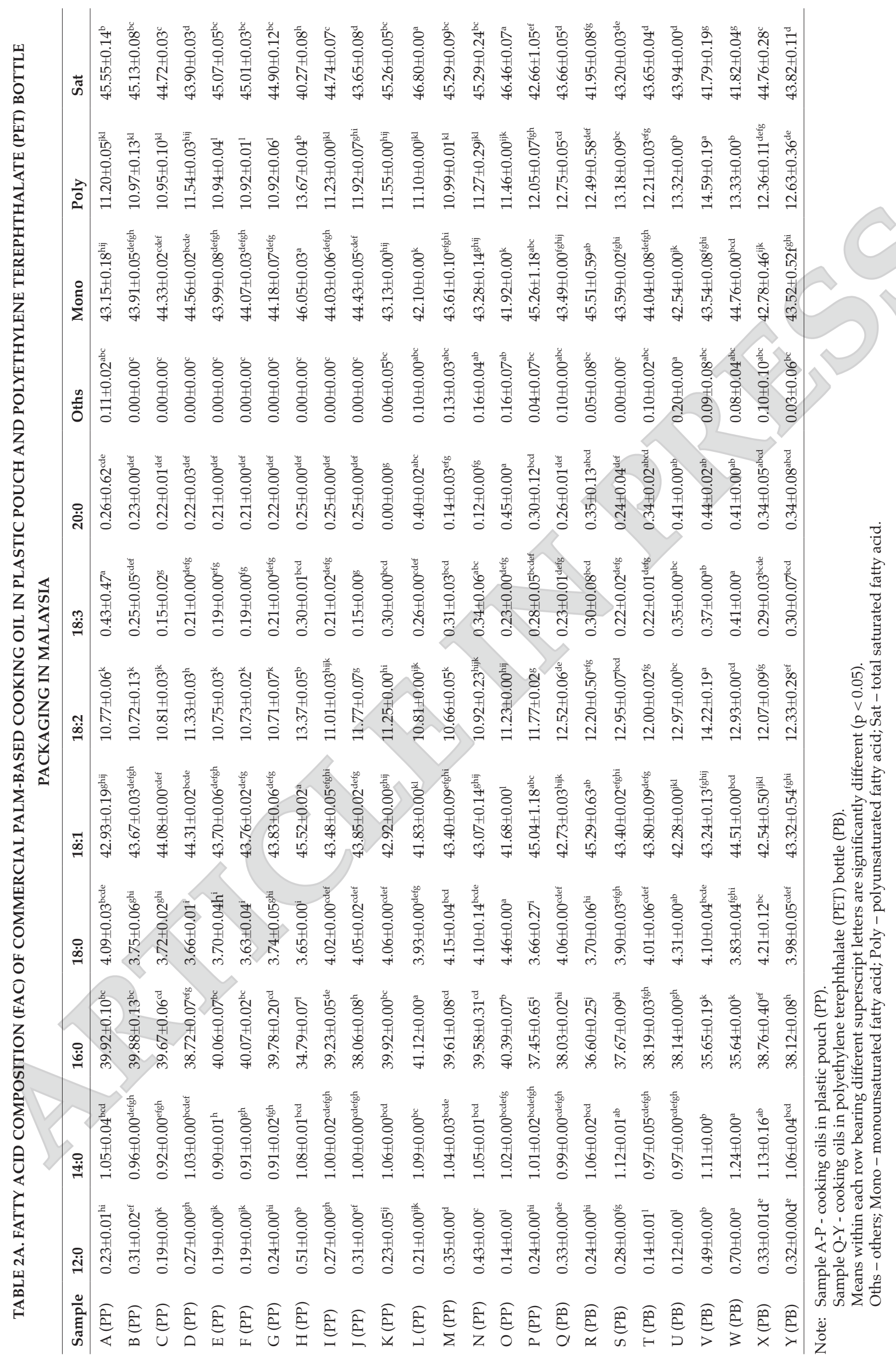


frying oil with high content of oleic acid (i.e. palm olein and sunflower oil) provides better health benefit. The linolenic acid in the palm cooking oil was well below $2 \%$ as higher linolenic acid imparts undesirable smell during frying and provide off-taste (bitter) to the fried product (Ahmad Tarmizi et al., 2014). According to Berger (2005) for industrial frying, linolenic acid value must be kept below 2\%. The palm cooking oils contained a balanced saturated and unsaturated fatty acids that provide stability to the oil. This makes palm cooking oil good for deep frying, as it is not potent to thermal degradation that normally takes place in unsaturated fatty acid (Ahmad Tarmizi et al., 2013).

According to a 2-sample t-test, the average monounsaturated fatty acid of cooking oils in both plastic pouch and PET bottle was not significantly different $(\mathrm{p}>0.05)$ and thus, were comparable (Table $2 b$ ). However, both average polyunsaturated and total saturated fatty acid of cooking oils in both plastic pouch and PET bottle were significantly different $\quad(p<0.05)$ Notwithstanding this, comparison of average total unsaturated (poly and mono- unsaturated) and total saturated fatty acids of cooking oils in both plastic pouch and PET bottle using 2-sample t-test were significantly different $(p<0.05)$. The finding showed that the average total unsaturated fatty acid in PET bottle cooking oil was higher (better) than those in plastic pouch. This result synchronised with the average saturated fatty acid findings when this fatty acid was lower (better) in PET bottle compared to those in the plastic pouch.

TABLE 2B. 2-SAMPLE T-TEST OF FATTY ACID COMPOSITION (FAC) GROUP OF COMMERCIAL PALMBASED COOKING OIL IN PLASTIC POUCH AND POLYETHYLENE TEREPHTHALATE (PET) BOTTLE PACKAGING IN MALAYSIA

\begin{tabular}{lccc}
\hline & \multicolumn{2}{c}{ Mean (\%) } & \\
\cline { 2 - 3 } FAC group & Plastic pouch & PET bottle & \\
& p-value \\
16:0 (Palmitic acid) & $39.29 \pm 1.47$ & $37.40 \pm 1.13$ & 0.00 \\
18:0 (Stearic acid) & $3.85 \pm 0.39$ & $4.01 \pm 0.18$ & 0.04 \\
18:1(Oleic acid) & $43.59 \pm 1.01$ & $43.40 \pm 1.02$ & 0.44 \\
18:2 (Linoleic acid) & $11.17 \pm 0.68$ & $12.70 \pm 0.70$ & 0.00 \\
Monounsaturated & $43.89 \pm 1.05$ & $43.70 \pm 0.97$ & 0.42 \\
Polyunsaturated & $11.42 \pm 0.69$ & $13.01 \pm 0.75$ & 0.00 \\
Total Unsaturated & $55.32 \pm 1.54$ & $56.71 \pm 1.07$ & 0.00 \\
Saturated & $44.64 \pm 1.51$ & $43.17 \pm 1.04$ & 0.00 \\
\hline
\end{tabular}

Note: $p$-value $<0.05$ indicates that there is significant different between cooking oil in plastic pouch and PET bottle.

\section{Triacylglycerols Composition}

The TAG composition of palm-based commercial cooking oils in Malaysia is shown in Tables $3 a, 3 b$ and $3 c$. TAG was also conducted to check the authenticity of the oil. However, TAG of cooking and frying oils is the least conducted, as FAC analysis is normally sufficient. Based on ANOVA, all of the commercial palm cooking oils' TAG were significantly different $(p<0.05)$ based on ANOVA. However, there were cooking oils that were not significantly different from each other. The cooking oils in plastic pouch and PET bottle contained diacylglycerols (DAG) of between $5.89 \%-7.45 \%$ and $5.92 \%-7.34 \%$, respectively. This made TAG of cooking oils in both plastic bottle and PET to be between $92.55 \%-94.11 \%$ and $92.66 \%-94.08 \%$, respectively. This is in line with the findings by Ahmad Tarmizi et al. (2015) reporting of about $93 \%$ TAG in his study. The amount of DAG contributes to higher level of polar compounds in palm cooking oil. POO, POP, PLO and PLP were the dominant TAG composition in these cooking oils. These TAG of cooking oil in plastic pouch were in the range of $26.18 \%-31.89 \%, 24.83 \%-28.66 \%$, $10.94 \%-14.08 \%$ and $9.53 \%-10.45 \%$, respectively while in PET bottle were in the range of $27.05 \%$ $30.88 \%, 21.12 \%-26.14 \%, 12.11 \%-13.48 \%$ and $10.10 \%-$ $11.66 \%$, respectively. This gave an overall range of between 26.18\%-31.89\%, 17.69\%-28.66\%, $10.94 \%$ $14.08 \%, 9.53 \%-11.66 \%$ of those TAG accordingly. This trend was similar to the palm olein TAG major composition in a study by Calliauw et al. (2007). In a study by Myat et al. (2009), it was found that the TAG composition of POO, POP, PLO and PLP of commercial palm olein from a supermarket in Kuala Lumpur was $31.69 \%, 26.89 \%, 14.43 \%$ and $10.14 \%$, respectively. These TAG were in the range of the analysed commercial palm cooking oil in this study, except for PLO in which the TAG was not in the range. The TAG found in trace amounts were PPP, MLP, OLL and SOS. It was interesting to note that all palm cooking oils in PET bottle has 0\% PPP while cooking oil in plastic pouch has PPP in the range of $0.00 \%-0.47 \%$. UUU (unsaturated-unsaturatedunsaturated), SUU (saturated-unsaturatedunsaturated), SUS (saturated-unsaturatedsaturated) and SSS (saturated-saturated-saturated) TAG of commercial palm cooking oils in plastic pouch in Malaysia ranged between 5.99\%-8.35\%, $42.68 \%-52.27 \%, 31.94 \%-44.77 \%$ and $0.00 \%-0.47 \%$, respectively while the range for cooking oils in PET bottle were $6.43 \%-9.15 \%, 44.65 \%-50.10 \%, 34.53 \%$ $42.64 \%$ and 0.00 , respectively. Based on ANOVA, UUU, SUU, SUS and SSS TAG of the commercial cooking oils in both plastic pouch and PET bottle were significantly different $(p<0.05)$. However, there were cooking oils that were not significantly different from each other. In addition, the average UUU, SUU and SUS TAG of the cooking oils in both 
TABLE 3A. TRIACYLGLYCEROL COMPOSITION (TAG) OF COMMERCIAL PALM COOKING OIL IN PLASTIC POUCH AND POLYETHYLENE TEREPHTHALATE (PET) BOTTLE PACKAGING IN MALAYSIA

\begin{tabular}{|c|c|c|c|c|c|c|c|}
\hline Sample & DAG & POO & POP & PLO & PLP & POS & OOO \\
\hline $\mathrm{A}(\mathrm{PP})$ & $6.44 \pm 0.04^{\text {defgh }}$ & $26.81 \pm 0.15^{\mathrm{hij}}$ & $27.68 \pm 0.00^{\mathrm{cd}}$ & $11.18 \pm 0.01^{\mathrm{jk}}$ & $9.86 \pm 0.09^{\mathrm{lmn}}$ & $5.10 \pm 0.05^{\mathrm{a}}$ & $4.28 \pm 0.05^{\mathrm{hi}}$ \\
\hline $\mathrm{B}(\mathrm{PP})$ & $6.11 \pm 0.15^{\mathrm{j} k}$ & $27.15 \pm 0.06^{\mathrm{gh}}$ & $27.30 \pm 0.08 \mathrm{f}^{\mathrm{g}}$ & $11.28 \pm 0.00^{\mathrm{hij}}$ & $9.96 \pm 0.04^{\mathrm{jklm}}$ & $4.82 \pm 0.05^{\mathrm{efg}}$ & $4.41 \pm 0.03^{\text {efgh }}$ \\
\hline $\mathrm{C}(\mathrm{PP})$ & $6.26 \pm 0.00^{\text {hij }}$ & $27.14 \pm 0.05^{\mathrm{gh}}$ & $27.19 \pm 0.04^{\mathrm{g}}$ & $11.56 \pm 0.01^{\mathrm{ghi}}$ & $9.66 \pm 0.02^{\text {no }}$ & $4.93 \pm 0.05^{\text {bcdef }}$ & $4.46 \pm 0.00^{\mathrm{ef}}$ \\
\hline $\mathrm{D}(\mathrm{PP})$ & $6.11 \pm 0.02^{\mathrm{jk}}$ & $28.66 \pm 0.02^{\mathrm{de}}$ & $24.83 \pm 0.05^{\mathfrak{j}}$ & $12.12 \pm 0.03^{\mathrm{f}}$ & $10.16 \pm 0.04^{\text {ghijk }}$ & $4.59 \pm 0.00^{\mathrm{h}}$ & $4.51 \pm 0.00^{\text {de }}$ \\
\hline $\mathrm{E}(\mathrm{PP})$ & $6.50 \pm 0.00^{\text {de }}$ & $26.99 \pm 0.13^{g h}$ & $27.45 \pm 0.11^{\operatorname{defg}}$ & $11.34 \pm 0.04^{\text {ghij }}$ & $9.90 \pm 0.01^{\mathrm{klmn}}$ & $4.84 \pm 0.03^{\text {efg }}$ & $4.26 \pm 0.08^{h i}$ \\
\hline $\mathrm{F}(\mathrm{PP})$ & $6.49 \pm 0.02^{\mathrm{def}}$ & $26.95 \pm 0.06^{\mathrm{ghi}}$ & $27.39 \pm 0.06^{\mathrm{efg}}$ & $11.39 \pm 0.14^{\text {ghij }}$ & $9.90 \pm 0.16^{\mathrm{klmn}}$ & $4.86 \pm 0.08^{\text {defg }}$ & $4.28 \pm 0.04^{\mathrm{hi}}$ \\
\hline $\mathrm{G}(\mathrm{PP})$ & $6.53 \pm 0.00^{\text {de }}$ & $27.07 \pm 0.10^{\mathrm{gh}}$ & $27.24 \pm 0.09^{\mathrm{fg}}$ & $11.36 \pm 0.06^{\text {ghij }}$ & $9.90 \pm 0.04^{\mathrm{klmn}}$ & $4.87 \pm 0.0$ & $4.34 \pm 0.02^{\text {fghi }}$ \\
\hline $\mathrm{H}(\mathrm{PP})$ & $7.45 \pm 0.01^{\mathrm{a}}$ & $31.89 \pm 0.01^{\mathrm{a}}$ & $17.69 \pm 0.03^{p}$ & $14.08 \pm 0.04^{\mathrm{a}}$ & $10.45 \pm 0.01^{\mathrm{def}}$ & 0 & $5.18 \pm 0.00^{\mathrm{a}}$ \\
\hline $\mathrm{I}(\mathrm{PP})$ & $6.31 \pm 0.00^{\text {fghi }}$ & $26.93 \pm 0.04^{\text {ghi }}$ & $27.85 \pm 0.08^{c}$ & $11.22 \pm 0.05^{\mathrm{j}}$ & $9.76 \pm 0$ & בחم م- & $4.28 \pm 0.04^{\mathrm{hi}}$ \\
\hline $\mathrm{J}(\mathrm{PP})$ & $6.35 \pm 0.02^{\text {efghi }}$ & $26.90 \pm 0.06^{\text {ghi }}$ & $26.80 \pm 0.00^{\mathrm{h}}$ & $11.60 \pm 0.02^{\mathrm{g}}$ & $9.53 \pm 0$ & $5.00 \pm 0.00^{\mathrm{abcd}}$ & $4.66 \pm 0.02^{d}$ \\
\hline $\mathrm{K}(\mathrm{PP})$ & $6.56 \pm 0.01^{\mathrm{d}}$ & $26.41 \pm 0.05^{\mathrm{jk}}$ & $27.51 \pm 0.13^{\text {def }}$ & $11.53 \pm 0.04^{\mathrm{ghi}}$ & $10.24 \pm 0.06^{\mathrm{fghi}}$ & $5.03 \pm 0.00^{\mathrm{ab}}$ & $3.93 \pm 0.10^{j}$ \\
\hline $\mathrm{L}(\mathrm{PP})$ & $6.36 \pm 0.04^{\text {efghi }}$ & $26.18 \pm 0.00^{\mathrm{k}}$ & $28.25 \pm 0.06^{\mathrm{b}}$ & $11.57 \pm$ & $10.30 \pm 0.08^{\text {efgh }}$ & $4.94 \pm 0.04^{\text {bcde }}$ & $3.86 \pm 0.02^{\mathrm{jk}}$ \\
\hline $\mathrm{M}(\mathrm{PP})$ & $6.41 \pm 0.07^{\text {defgh }}$ & $27.20 \pm 0.11^{\mathrm{gh}}$ & $27.67 \pm 0.04^{\mathrm{cd}}$ & $10.94 \pm 0$ & $9.69 \pm 0.10^{\mathrm{mno}}$ & $4.87 \pm 0.06^{\text {cdefg }}$ & $4.45 \pm 0.01^{\mathrm{efg}}$ \\
\hline $\mathrm{N}(\mathrm{PP})$ & $5.89 \pm 0.00^{1}$ & $27.24 \pm 0.07^{\mathrm{g}}$ & $27.63 \pm 0.03^{\text {cde }}$ & & $9.97 \pm 0.00^{\mathrm{ijklm}}$ & $4.85 \pm 0.04^{\mathrm{efg}}$ & $4.35 \pm 0.02^{\text {fghi }}$ \\
\hline $\mathrm{O}(\mathrm{PP})$ & $6.18 \pm 0.02^{\mathrm{ij}}$ & $26.26 \pm 0.08^{\mathrm{k}}$ & $28.66 \pm 0.0$ & $11.58 \pm 0$ & $10.38 \pm 0.11^{\text {defg }}$ & $4.82 \pm 0.03^{\mathrm{efg}}$ & $3.74 \pm 0.01^{\mathrm{k}}$ \\
\hline $\mathrm{P}(\mathrm{PP})$ & $6.39 \pm 0.05^{\text {defgh }}$ & $26.55 \pm 0.11^{\mathrm{ijk}}$ & 7761 & $11.46 \pm 0.06^{\text {ghij }}$ & $10.21 \pm 0.04^{\text {fghij }}$ & $5.00 \pm 0.00^{\mathrm{abc}}$ & $3.98 \pm 0.01^{j}$ \\
\hline $\mathrm{Q}(\mathrm{PB})$ & $5.96 \pm 0.06^{\mathrm{kl}}$ & $27.05 \pm 0.11^{\mathrm{gh}}$ & & $12.11 \pm 0.15^{\mathrm{f}}$ & $10.65 \pm 0.06^{\mathrm{cd}}$ & $4.76 \pm 0.00^{g}$ & $4.19 \pm 0.03^{\mathrm{i}}$ \\
\hline $\mathrm{R}(\mathrm{PB})$ & $6.27 \pm 0.07^{\text {ghij }}$ & & & $13.06 \pm 0.02^{\mathrm{cd}}$ & $10.10 \pm 0.12^{\mathrm{hijkl}}$ & $3.88 \pm 0.02^{k}$ & $5.01 \pm 0.06^{\mathrm{b}}$ \\
\hline$S(P B)$ & $7.18 \pm 0.01^{\mathrm{b}}$ & $29.02 \pm 0.25^{\mathrm{d}}$ & $21.12 \pm 0.00^{\mathrm{mn}}$ & $13.07 \pm 0.04^{\mathrm{cd}}$ & $11.66 \pm 0.12^{a}$ & $3.93 \pm 0.03^{k}$ & $4.34 \pm 0.02^{\text {fghi }}$ \\
\hline $\mathrm{T}(\mathrm{PB})$ & $6.46 \pm 0.04^{\operatorname{defg}}$ & 28.3 & $24.53 \pm 0.13^{\mathrm{k}}$ & $12.61 \pm 0.01^{\mathrm{e}}$ & $10.38 \pm 0.03^{\operatorname{defg}}$ & $4.36 \pm 0.03^{j}$ & $4.30 \pm 0.00^{\mathrm{ghi}}$ \\
\hline $\mathrm{U}(\mathrm{PB})$ & 6.43 & $27.05 \pm 0.00^{g h}$ & $24.42 \pm 0.50^{\mathrm{k}}$ & $12.94 \pm 0.10^{\mathrm{d}}$ & $11.23 \pm 0.04^{\mathrm{b}}$ & $4.51 \pm 0.06^{\mathrm{hi}}$ & $3.89 \pm 0.03^{\mathrm{jk}}$ \\
\hline $\mathrm{V}(\mathrm{PB})$ & $0.02^{b}$ & $27.98 \pm 0.01^{\mathrm{f}}$ & $21.01 \pm 0.04^{\mathrm{n}}$ & $13.32 \pm 0.10^{\mathrm{bc}}$ & $10.56 \pm 0.04^{\text {cde }}$ & $3.71 \pm 0.01^{1}$ & $4.83 \pm 0.00^{c}$ \\
\hline $\mathrm{W}(\mathrm{PB})$ & $34 \pm 0.06^{\mathrm{ab}}$ & $30.35 \pm 0.06^{c}$ & $19.84 \pm 0.00^{\circ}$ & $13.48 \pm 0.16^{\mathrm{b}}$ & $10.42 \pm 0.05^{\operatorname{defg}}$ & $3.37 \pm 0.00^{\mathrm{m}}$ & $4.91 \pm 0.06^{\mathrm{bc}}$ \\
\hline $\mathrm{X}(\mathrm{PB})$ & $5.92 \pm 0.07^{\mathrm{kl}}$ & $27.10 \pm 0.22^{\mathrm{gh}}$ & $25.96 \pm 0.04^{\mathrm{i}}$ & $12.11 \pm 0.13^{f}$ & $10.60 \pm 0.07^{\mathrm{cd}}$ & $4.79 \pm 0.05^{\mathrm{fg}}$ & $4.27 \pm 0.02^{\mathrm{hi}}$ \\
\hline $\mathrm{Y}(\mathrm{PB})$ & $6.77 \pm 0.05^{c}$ & $28.43 \pm 0.14^{\mathrm{e}}$ & $23.50 \pm 0.03^{1}$ & $12.46 \pm 0.10^{\mathrm{e}}$ & $10.82 \pm 0.05^{c}$ & $4.38 \pm 0.01^{\mathrm{ij}}$ & $4.37 \pm 0.04^{\text {efgh }}$ \\
\hline
\end{tabular}

Note: Sample A - P - cooking oils in plastic pouch (PP).

Sample Q - Y - cooking oils in polyethylene terephthalate (PET) bottle (PB).

Means within each row bearing different superscript letters are significantly different $(\mathrm{p}<0.05)$.

DAG - diacylglycerols; M - myristic; P - palmitic; S - stearic; O - oleic; L - linoleic. 
TABLE 3B. TRIACYLGLYCEROL COMPOSITION (TAG) OF COMMERCIAL PALM COOKING OIL IN PLASTIC POUCH AND POLYETHYLENE TEREPHTHALATE (PET) BOTTLE PACKAGING IN MALAYSIA (con't)

\begin{tabular}{|c|c|c|c|c|c|c|c|}
\hline Sample & SOO & PLL & OLO & sos & OLL & MLP & PPP \\
\hline $\mathrm{A}(\mathrm{PP})$ & $2.56 \pm 0.04^{\text {cde }}$ & $2.46 \pm 0.03^{\mathrm{h}}$ & $2.00 \pm 0.02^{\text {hijklmn }}$ & $0.59 \pm 0.00^{b c}$ & $0.49 \pm 0.00^{\text {defgh }}$ & $0.36 \pm 0.00^{\text {efghij }}$ & $0.23 \pm 0.00^{e}$ \\
\hline $\mathrm{B}(\mathrm{PP})$ & $2.61 \pm 0.00^{\text {cde }}$ & $2.66 \pm 0.06^{\mathrm{fgh}}$ & $2.01 \pm 0.00^{\text {hijkl }}$ & $0.61 \pm 0.00^{\mathrm{abc}}$ & $0.49 \pm 0.02^{\text {efgh }}$ & $0.40 \pm 0.02^{\text {bcdefg }}$ & $0.24 \pm 0.00 \mathrm{~d}^{\mathrm{e}}$ \\
\hline $\mathrm{C}(\mathrm{PP})$ & $2.62 \pm 0.00^{\text {cde }}$ & $2.53 \pm 0.01^{\mathrm{gh}}$ & $2.03 \pm 0.02^{h i j}$ & $0.64 \pm 0.02^{\mathrm{ab}}$ & $0.47 \pm 0.01^{\text {efgh }}$ & $0.31 \pm 0.00^{\mathrm{jk}}$ & $0.24 \pm 0.00^{\text {de }}$ \\
\hline $\mathrm{D}(\mathrm{PP})$ & $2.65 \pm 0.02^{\mathrm{cd}}$ & $2.72 \pm 0.04^{\mathrm{efg}}$ & $2.06 \pm 0.00^{\mathrm{ghi}}$ & $0.61 \pm 0.04^{\mathrm{abc}}$ & $0.48 \pm 0.00^{\text {efgh }}$ & $0.39 \pm 0.00^{\text {cdefgh }}$ & $0.16 \pm 0.02^{f}$ \\
\hline $\mathrm{E}(\mathrm{PP})$ & $2.57 \pm 0.04^{\text {cde }}$ & $2.52 \pm 0.05^{\mathrm{gh}}$ & $1.98 \pm 0.01^{\mathrm{ijklmn}}$ & $0.59 \pm 0.00^{\mathrm{bcd}}$ & $0.46 \pm 0.01^{\mathrm{fgh}}$ & $0.35 \pm 0.00^{\text {ghij }}$ & $\pm 0.02^{c}$ \\
\hline $\mathrm{F}(\mathrm{PP})$ & $2.48 \pm 0.02^{\text {efg }}$ & $2.61 \pm 0.08^{g h}$ & $1.98 \pm 0.04^{\mathrm{ijklmn}}$ & $0.59 \pm 0.00^{\mathrm{bcd}}$ & $0.50 \pm 0.00^{\text {defgh }}$ & $0.35 \pm 0.0$ & $.01^{\mathrm{cd}}$ \\
\hline $\mathrm{G}(\mathrm{PP})$ & $2.54 \pm 0.01^{\text {cdef }}$ & $2.51 \pm 0.03^{\mathrm{gh}}$ & $2.01 \pm 0.04^{\mathrm{hijklm}}$ & $0.60 \pm 0.01^{\mathrm{bc}}$ & $0.47 \pm 0.03^{\text {efgh }}$ & & $00^{\text {cde }}$ \\
\hline $\mathrm{H}(\mathrm{PP})$ & $3.00 \pm 0.01^{\mathrm{a}}$ & $3.32 \pm 0.04^{\mathrm{ab}}$ & $2.51 \pm 0.00^{\mathrm{b}}$ & $0.38 \pm 0.02^{f}$ & $0.66 \pm 0.02^{c}$ & $2^{a b c}$ & $.00 \pm 0.00^{g}$ \\
\hline $\mathrm{I}(\mathrm{PP})$ & $2.54 \pm 0.01^{\text {cdef }}$ & $2.46 \pm 0.02^{\mathrm{h}}$ & $1.96 \pm 0.00^{\mathrm{jklmn}}$ & $0.62 \pm 0.00^{\mathrm{abc}}$ & $0.45 \pm 0.01^{\mathrm{gh}}$ & $0.34 \pm 0.00^{\text {hij }}$ & $0.29 \pm 0.00^{c}$ \\
\hline $\mathrm{J}(\mathrm{PP})$ & $2.62 \pm 0.01^{\text {cde }}$ & $2.47 \pm 0.08^{\mathrm{h}}$ & $2.21 \pm 0.03^{\text {de }}$ & $0.60 \pm 0.00^{\mathrm{bc}}$ & $0.82 \pm 0.03^{b}$ & $0.26 \pm 0.02^{k}$ & $0.22 \pm 0.00^{\mathrm{e}}$ \\
\hline $\mathrm{K}(\mathrm{PP})$ & $2.48 \pm 0.08^{\mathrm{efg}}$ & $2.56 \pm 0.07^{\mathrm{gh}}$ & $1.91 \pm 0.01^{\mathrm{mn}}$ & $0.61 \pm 0.02^{\text {bc }}$ & $0.43 \pm 0.02^{\mathrm{h}}$ & $0.36 \pm 0.00^{\text {fghij }}$ & $0.47 \pm 0.03^{\mathrm{a}}$ \\
\hline $\mathrm{L}(\mathrm{PP})$ & $2.37 \pm 0.04^{g}$ & $2.57 \pm 0.03^{g h}$ & $1.92 \pm 0.01^{1 \mathrm{mn}}$ & $0.61 \pm 0.03^{\mathrm{abc}}$ & $0.45 \pm 0.02^{g h}$ & $0.33 \pm 0.00^{\mathrm{ij}}$ & $0.33 \pm 0.00^{\mathrm{b}}$ \\
\hline $\mathrm{M}(\mathrm{PP})$ & $2.59 \pm 0.08^{\text {cde }}$ & $2.51 \pm 0.06^{\mathrm{gh}}$ & $1.93 \pm 0.02^{\mathrm{klmn}}$ & & $0.49 \pm 0.04^{\mathrm{efgh}}$ & $0.42 \pm 0.04^{\text {bcde }}$ & $0.26 \pm 0.00^{\text {cde }}$ \\
\hline $\mathrm{N}(\mathrm{PP})$ & $2.52 \pm 0.08^{\mathrm{def}}$ & $2.59 \pm 0.00^{g h}$ & $2.00 \pm 0.0$ & $0.60 \pm 0.00^{\mathrm{bc}}$ & $0.52 \pm 0.02^{\text {defg }}$ & $0.39 \pm 0.00^{\text {cdefgh }}$ & $0.23 \pm 0.01^{\mathrm{de}}$ \\
\hline $\mathrm{O}(\mathrm{PP})$ & $2.40 \pm 0.04^{\mathrm{fg}}$ & $2.61 \pm 0.09^{g h}$ & $1.81 \pm 0.0$ & $0.57 \pm 0.03^{\text {bcde }}$ & $0.44 \pm 0.00^{\mathrm{gh}}$ & $0.34 \pm 0.02^{h i j}$ & $0.24 \pm 0.00^{\mathrm{de}}$ \\
\hline $\mathrm{P}(\mathrm{PP})$ & $2.54 \pm 0.01^{\text {cdef }}$ & & & $0.58 \pm 0.00^{\text {bcde }}$ & $0.48 \pm 0.03^{\text {efgh }}$ & $0.34 \pm 0.00^{\text {hij }}$ & $0.44 \pm 0.00^{\mathrm{a}}$ \\
\hline $\mathrm{Q}(\mathrm{PB})$ & $2.55 \pm 0.04^{\text {cde }}$ & $2.94 \pm 0.04^{\text {cde }}$ & $2.02 \pm 0.06^{\text {hijk }}$ & $0.70 \pm 0.03^{\mathrm{a}}$ & $0.55 \pm 0.00^{\mathrm{def}}$ & $0.40 \pm 0.00^{\text {bcdefg }}$ & $0.00 \pm 0.00^{\mathrm{g}}$ \\
\hline $\mathrm{R}(\mathrm{PB})$ & $2.87 \pm 0.01^{\mathrm{ab}}$ & $.08^{\text {def }}$ & $2.26 \pm 0.00^{\mathrm{d}}$ & $0.53 \pm 0.02^{\text {cde }}$ & $0.51 \pm 0.01^{\text {defgh }}$ & $0.43 \pm 0.00^{\mathrm{abc}}$ & $0.00 \pm 0.00^{g}$ \\
\hline $\mathrm{S}(\mathrm{PB})$ & $2.83 \pm 0.02^{\mathrm{b}}$ & $3.14 \pm 0.04^{\mathrm{bc}}$ & $2.13 \pm 0.00^{\text {efg }}$ & $0.55 \pm 0.03^{\text {bcde }}$ & $0.58 \pm 0.00^{\text {cd }}$ & $0.48 \pm 0.00^{a}$ & $0.00 \pm 0.00^{\mathrm{g}}$ \\
\hline $\mathrm{T}(\mathrm{PB})$ & & $2.91 \pm 0.06^{\mathrm{de}}$ & $2.00 \pm 0.03^{\text {hijklm }}$ & $0.56 \pm 0.04^{\text {bcde }}$ & $0.50 \pm 0.05^{\text {defgh }}$ & $0.37 \pm 0.02^{\text {defghi }}$ & $0.00 \pm 0.00^{\mathrm{g}}$ \\
\hline $\mathrm{U}(\mathrm{PB})$ & $2.59 \pm 0.01^{\text {cde }}$ & $3.36 \pm 0.00^{\mathrm{ab}}$ & $2.00 \pm 0.01^{\text {hijklmn }}$ & $0.61 \pm 0.01^{\mathrm{abc}}$ & $0.55 \pm 0.01^{\text {de }}$ & $0.45 \pm 0.01^{\mathrm{ab}}$ & $0.00 \pm 0.00^{g}$ \\
\hline $\mathrm{V}(\mathrm{PB})$ & $2.68 \pm 0.02^{c}$ & $3.52 \pm 0.08^{a}$ & $2.90 \pm 0.02^{\mathrm{a}}$ & $0.49 \pm 0.05^{\mathrm{e}}$ & $1.43 \pm 0.03^{\mathrm{a}}$ & $0.42 \pm 0.01^{\mathrm{bcd}}$ & $0.00 \pm 0.00^{\mathrm{g}}$ \\
\hline $\mathrm{W}(\mathrm{PB})$ & $2.88 \pm 0.00^{\mathrm{ab}}$ & $3.39 \pm 0.11^{\mathrm{a}}$ & $2.38 \pm 0.01^{c}$ & $0.50 \pm 0.04^{\text {de }}$ & $0.76 \pm 0.00^{\mathrm{b}}$ & $0.41 \pm 0.01^{\text {bcdef }}$ & $0.00 \pm 0.00^{\mathrm{g}}$ \\
\hline $\mathrm{X}(\mathrm{PB})$ & $2.54 \pm 0.03^{\text {cdef }}$ & $2.94 \pm 0.02^{\text {cde }}$ & $2.17 \pm 0.01^{\text {def }}$ & $0.59 \pm 0.02^{\mathrm{bcd}}$ & $0.66 \pm 0.04^{c}$ & $0.39 \pm 0.00^{\text {cdefgh }}$ & $0.00 \pm 0.00^{\mathrm{g}}$ \\
\hline $\mathrm{Y}(\mathrm{PB})$ & $2.65 \pm 0.02^{\mathrm{cd}}$ & $2.97 \pm 0.02^{\mathrm{cd}}$ & $2.08 \pm .01^{\mathrm{fgh}}$ & $0.60 \pm 0.01^{\mathrm{bc}}$ & $0.55 \pm 0.00^{\text {de }}$ & $0.45 \pm 0.00^{\mathrm{ab}}$ & $0.00 \pm 0.00^{g}$ \\
\hline
\end{tabular}

Note: Sample A - P - cooking oils in plastic pouch (PP).

Sample Q - Y - cooking oils in polyethylene terephthalate (PET) bottle (PB).

Means within each row bearing different superscript letters are significantly different $(\mathrm{p}<0.05)$.

M - myristic; P - palmitic; S - stearic; O - oleic; L - linoleic. 
TABLE 3C. TRIACYLGLYCEROL COMPOSITION (TAG) GROUPING OF COMMERCIAL PALM-BASED COOKING OIL IN PLASTIC POUCH AND POLYETHYLENE TEREPHTHALATE (PET) BOTTLE PACKAGING IN MALAYSIA

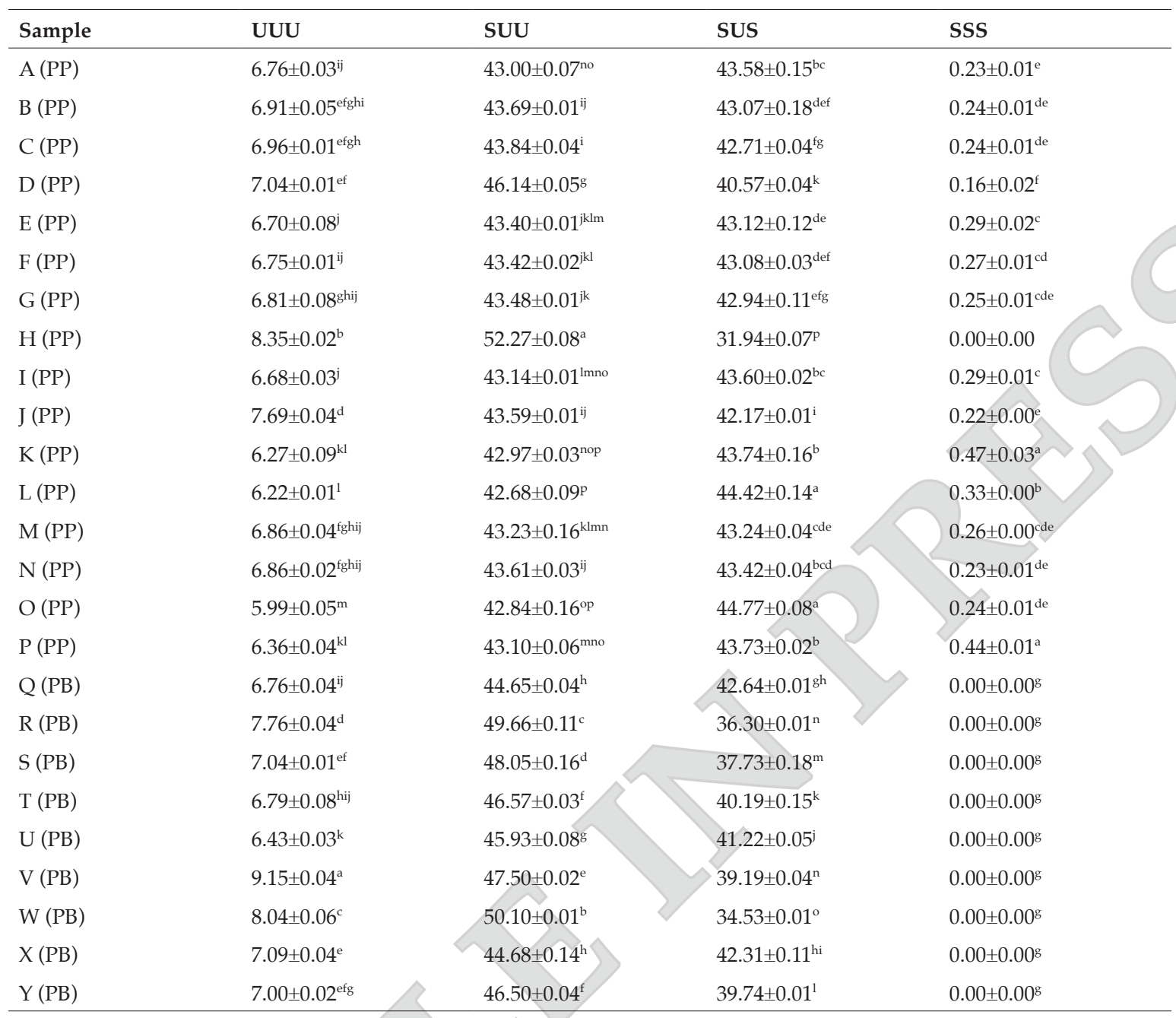

Note: Sample A-P - cooking oils in plastic pouch (PP).

Sample Q-Y - cooking oils in polyethylene terephthalate (PET) bottle (PB).

UUU - unsaturated-unsaturated-unsaturated.

SUU - saturated-unsaturated-unsaturated

SUS - saturated-unsaturated-saturated.

SSS - saturated-saturated-saturated.

plastic pouch and PET bottle were significantly different $(\mathrm{p}<0.05)$ too based on 2-sample t-test (Table 3d). Average UUU and SUU TAG of cooking oil in plastic bottle were higher (better) than those in plastic pouch. However, the average SUS TAG of cooking oil in PET bottle was lower (better) than those in plastic pouch. The 2-sample t-test for average SSS cannot be conducted as PET bottle has similar average SSS value.

\section{Vitamin E}

Vitamin E consist of tocopherols and tocotrienols isomers that is fat soluble having potent anti-oxidative and other healthpromoting properties (Sundram et al., 2003). Good dietary sources of vitamin $\mathrm{E}$ include vegetable oils and nuts, such as almonds, peanuts and hazelnuts.
TABLE 3D. 2-SAMPLE T-TEST OF TRIACYLGLYCEROL COMPOSITION (TAG) GROUP OF COMMERCIAL PALM-

BASED COOKING OIL IN PLASTIC POUCH AND POLYETHYLENE TEREPHTHALATE (PET) BOTTLE PACKAGING IN MALAYSIA

\begin{tabular}{cccc}
\hline \multirow{2}{*}{ TAG group } & \multicolumn{2}{c}{ Mean $(\%)$} & p-value \\
\cline { 2 - 3 } & Plastic pouch & PET bottle & \\
\hline UUU & $6.82 \pm 0.56$ & $7.34 \pm 0.82$ & 0.03 \\
SUU & $44.02 \pm 2.30$ & $47.07 \pm 1.89$ & 0.00 \\
SUS & $52.50 \pm 2.92$ & $38.98 \pm 2.83$ & 0.00 \\
SSS & - & - & - \\
\hline
\end{tabular}

Note: $p$-value $<0.05$ indicates that there is significant different between cooking oil in plastic pouch and PET bottle.

UUU - unsaturated-unsaturated-unsaturated.

SUU - saturated-unsaturated-unsaturated.

SUS - saturated-unsaturated-saturated.

SSS - saturated-saturated-saturated. 
Palm oil is rich in vitamin E (600-1000 ppm in crude palm oil). Palm oil's vitamin $\mathrm{E}$ is rich in tocotrienols (75\%-80\% of the total vitamin E) which seldom found in vegetable oil ( $\mathrm{Ng}$ et al., 2004; Choo et al., 1996). The vitamin E provides oxidative stability to the oil (Sundram et al., 2003) as well as during heating and frying by decelerating oxidative degradation (Matthäus et al., 2009). Many studies suggested that tocotrienols is a more potent antioxidant compared to tocopherols (i.e. Kamar et al., 1997). Many studies revealed that palm vitamin E demonstrates numerous health-benefits besides anti-oxidant. This includes cardioprotective, nephroprotective, neuroprotective, hepatoprotective, anti-cancer, anti-diabetic, cancer-suppression properties and bone metabolism positive effect (Siddiqui et al., 2013; Norazlina et al., 2010; Sen et al., 2010; Siddiqui et al., 2010; Azlina et al., 2005; Mutalib et al., 2003; Nesaretnam et al., 1998; Goh et al., 1994; Serbinova and Packer, 1994; Nesaretnam et al., 1992; Ngah et al., 1991).

Total vitamin E, total tocotrienols and total tocopherols of cooking oils in the plastic pouch were in the range of 539.17-949.16 ppm, 443.58$640.21 \mathrm{ppm}$ and 177.99-269.14 ppm, accordingly (Table $4 a$ ). The total vitamin $\mathrm{E}$, total tocotrienols and total tocopherols of cooking oils in the PET bottle on the other hand were in the range of 498.02-856.00 ppm, 364.26-705.11 ppm and 133.76235.65 ppm respectively. The overall range of total vitamin E, total tocotrienols and total tocopherols in commercial cooking oil (plastic pouch and PET bottle) were 498.02-949.16 ppm, 364.26-640.21 ppm and 133.76-269.14 ppm, respectively and were significantly different $(\mathrm{p}<0.05)$ based on ANOVA. However, there were cooking oils that were not significantly different from each other. Vitamin $\mathrm{E}$ in RBD palm olein was 561 ppm as reported by Nagendran et al. (2000). Sundram et al. (2001), on the other hand, disclosed that vitamin $\mathrm{E}$ in RBD palm olein was 824.3 ppm with $196.6 \mathrm{ppm}$ of tocopherol and $627.7 \mathrm{ppm}$ of tocotrienol. The level of tocotrienols decreases with refining when compared with crude palm oil. Sample E (plastic pouch) has the highest amount of total vitamin $\mathrm{E}$ and tocopherols while sample A (plastic pouch) has the highest total tocotrienols. Sample Q (PET bottle) has the lowest amount of total vitamin $\mathrm{E}$, tocotrienols and tocopherols. The total vitamin E of sample Q (498.02 ppm) is quite low if were to compare with the study by Sundram and Noor (2001). Apart from sample $\mathrm{Q}$, other commercial cooking oils have considerable total vitamin E ranging from 539.17-949.19 ppm. Based on 2-sample t-test, $\mathrm{p}<0.05$ was obtained for the average total tocopherols for cooking oils in both plastic pouch and PET bottle packagings which indicated that these packagings were significantly different with plastic pouch having higher (better) total tocopherols (Table 4b). However, average total
TABLE 4A. TOTAL TOCOLS COMPOSITION OF COMMERCIAL PALM-BASED COOKING OIL IN PLASTIC
POUCH AND POLYETHYLENE TEREPHTHALATE (PET) BOTTLE PACKAGING IN MALAYSIA

\begin{tabular}{|c|c|c|c|}
\hline Sample & $\begin{array}{c}\text { Total } \\
\text { tocopherols } \\
(\mathrm{ppm})\end{array}$ & $\begin{array}{c}\text { Total } \\
\text { tocotrienols } \\
(\mathrm{ppm})\end{array}$ & $\begin{array}{l}\text { Total Vitamin E } \\
\text { (tocols) (ppm) }\end{array}$ \\
\hline $\mathrm{A}(\mathrm{PP})$ & $235.91 \pm 4.07^{\mathrm{b}}$ & $640.21 \pm 11.31^{\mathrm{b}}$ & $876.12 \pm 15.38^{\mathrm{b}}$ \\
\hline $\mathrm{B}(\mathrm{PP})$ & $230.49 \pm 0.64^{\mathrm{bcd}}$ & $603.06 \pm 1.13^{\mathrm{de}}$ & $833.55 \pm 1.77^{\text {cdef }}$ \\
\hline $\mathrm{C}(\mathrm{PP})$ & $234.60 \pm 4.20^{\mathrm{bc}}$ & $617.92 \pm 11.19^{\text {cde }}$ & $852.52 \pm 15.39^{\mathrm{bcd}}$ \\
\hline $\mathrm{D}(\mathrm{PP})$ & $231.29 \pm 3.75^{\mathrm{bcd}}$ & $583.94 \pm 10.65^{\text {efg }}$ & $815.23 \pm 14.39^{\text {defg }}$ \\
\hline $\mathrm{E}(\mathrm{PP})$ & $269.14 \pm 6.55^{\mathrm{a}}$ & $608.01 \pm 16.23^{a}$ & $949.16 \pm 22.78^{\mathrm{a}}$ \\
\hline $\mathrm{F}(\mathrm{PP})$ & $226.41 \pm 1.70^{\text {bcde }}$ & $580.80 \pm 3.58^{\mathrm{efg}}$ & $807.22 \pm 5.28^{\mathrm{efg}}$ \\
\hline $\mathrm{G}(\mathrm{PP})$ & $195.59 \pm 2.81^{\mathrm{ij}}$ & $443.58 \pm 6.56^{\circ}$ & $539.17 \pm 9.36^{\mathrm{m}}$ \\
\hline $\mathrm{H}(\mathrm{PP})$ & $221.77 \pm 5.59^{\mathrm{def}}$ & $540.99 \pm 13.65^{\text {hijkl }}$ & $762.77 \pm 19.23^{\mathrm{hi}}$ \\
\hline $\mathrm{I}(\mathrm{PP})$ & $213.20 \pm 3.58^{\mathrm{fg}}$ & $569.95 \pm 9.30^{\mathrm{fgh}}$ & $783.15 \pm 12.87^{\mathrm{gh}}$ \\
\hline $\mathrm{J}(\mathrm{PP})$ & $230.68 \pm 3.97^{\text {bed }}$ & $556.93 \pm 9.64^{\text {ghijk }}$ & $787.61 \pm 13.61^{\mathrm{gh}}$ \\
\hline $\mathrm{K}(\mathrm{PP})$ & $200.04 \pm 1.26^{\mathrm{hi}}$ & $533.98 \pm 3.46^{\mathrm{jlm}}$ & $734.02 \pm 4.47^{\mathrm{ij}}$ \\
\hline $\mathrm{L}(\mathrm{PP})$ & $197.52 \pm 5.97^{\mathrm{hi}}$ & $530.71 \pm 2.63^{\mathrm{km}}$ & $728.23 \pm 7.95^{\mathrm{ijk}}$ \\
\hline $\mathrm{M}(\mathrm{PP})$ & 228.8 & $564.57 \pm 8.73^{\text {fghi }}$ & $793.41 \pm 11.57^{\mathrm{fgh}}$ \\
\hline $\mathrm{N}(\mathrm{PP})$ & $236.95 \pm 1.77^{\mathrm{b}}$ & $582.25 \pm 4.71^{\text {efg }}$ & $819.20 \pm 6.46^{\text {cdefg }}$ \\
\hline $\mathrm{O}(\mathrm{PP})$ & $177.99 \pm 2.47^{\mathrm{kl}}$ & $509.80 \pm 7.04^{\mathrm{mn}}$ & $687.79 \pm 9.51^{\mathrm{kl}}$ \\
\hline $\mathrm{P}(\mathrm{PP})$ & $208.97 \pm 4.48^{\mathrm{gh}}$ & $520.74 \pm 12.29^{\mathrm{Im}}$ & $729.71 \pm 16.77^{\mathrm{ij}}$ \\
\hline $\mathrm{Q}(\mathrm{PB})$ & $133.76 \pm 1.51^{\mathrm{m}}$ & $364.26 \pm 4.45^{\mathrm{p}}$ & $498.02 \pm 5.95^{n}$ \\
\hline $\mathrm{R}(\mathrm{PB})$ & $216.83 \pm 5.13^{\text {efg }}$ & $639.18 \pm 15.67^{\mathrm{bc}}$ & $856.00 \pm 20.79^{\mathrm{bc}}$ \\
\hline $\mathrm{S}(\mathrm{PB})$ & $198.32 \pm 1.39^{\mathrm{hi}}$ & $560.97 \pm 3.96^{\text {ghij }}$ & $759.29 \pm 5.27^{\mathrm{hij}}$ \\
\hline $\mathrm{T}(\mathrm{PB})$ & $169.36 \pm 3.22^{1}$ & $484.77 \pm 6.66^{\mathrm{n}}$ & $654.13 \pm 9.88^{\mathrm{lm}}$ \\
\hline $\mathrm{U}(\mathrm{PB})$ & $184.95 \pm 1.74^{\mathrm{jk}}$ & $535.93 \pm 5.44^{\mathrm{ijklm}}$ & $720.88 \pm 7.13^{\mathrm{jk}}$ \\
\hline $\mathrm{V}(\mathrm{PB})$ & $216.89 \pm 1.72^{\text {efg }}$ & $705.11 \pm 7.47^{\mathrm{a}}$ & $922.00 \pm 9.06^{\mathrm{a}}$ \\
\hline $\mathrm{W}(\mathrm{PB})$ & $235.65 \pm 5.68^{\mathrm{b}}$ & $585.91 \pm 13.72^{\text {efg }}$ & $821.56 \pm 19.39^{\text {cdefg }}$ \\
\hline $\mathrm{X}(\mathrm{PB})$ & $233.72 \pm 1.76^{\mathrm{bc}}$ & $610.02 \pm 6.10^{\text {cde }}$ & $843.74 \pm 7.84^{\text {bcde }}$ \\
\hline $\mathrm{Y}(\mathrm{PB})$ & $233.40 \pm 4.49^{\text {cdef }}$ & $592.89 \pm 13.74^{\text {def }}$ & $816.29 \pm 18.23^{\text {cdefg }}$ \\
\hline
\end{tabular}

Note: Sample A-P - cooking oils in plastic pouch (PP).

Sample Q-Y - cooking oils in polyethylene terephthalate (PET) bottle (PB).

Means within each row bearing different superscript letters are significantly different $(\mathrm{p}<0.05)$.

TABLE 4B. 2-SAMPLE T-TEST OF TOTAL TOCOLS COMPOSITION OF COMMERCIAL PALM-BASED COOKING OIL IN PLASTIC POUCH AND POLYETHYLENE TEREPHTHALATE (PET) BOTTLE PACKAGING IN MALAYSIA

\begin{tabular}{lccc}
\hline \multirow{2}{*}{ Tocols } & \multicolumn{2}{c}{ Mean $(\mathrm{ppm})$} & \\
\cline { 2 - 3 } & Plastic pouch & PET bottle & p-value \\
\hline Tocopherols & $221.20 \pm 21.50$ & $201.40 \pm 32.50$ & 0.01 \\
Tocotrienols & $566.2 \pm 55.10$ & $564.30 \pm 94.00$ & 0.93 \\
Vitamin E & $787.40 \pm 74.3$ & $766.00 \pm 123.00$ & 0.41 \\
\hline
\end{tabular}

Note: $p$-value $<0.05$ indicates that there is significant different between cooking oil in plastic pouch and PET bottle. 
vitamin E (tocols) and total tocotrienols of cooking oils in both packaging were insignificantly different ( $p>0.05)$ and thus were comparable.

\section{Iodine Value}

IV is a measure of degree of unsaturation of oils and fats. The higher the IV, the higher the unsaturated fatty acids content of the oils and fats (Yousefi et al., 2013) which resulted to higher incident of oxidation (Nawal et al., 2014) and frequency of oil discarding (Orthoefer and List, 2007). IV of cooking oils in both plastic pouch and PET bottle collected were ranged from $55.50 \%-63.44 \%$ and $58.33 \%-62.89 \%$, respectively (Table $5 a$ ). Based on ANOVA, the IV of cooking oils in both packagings were significantly different $(\mathrm{p}<0.05)$ resulting to overall IV ranging from 55.5063.44. However, there were cooking oils that were not significantly different from each other. The table also showed that most cooking oils in PET bottles have higher IV compared to cooking oils in pouches, except for sample H. Malaysian Standard for Palm Olein - MS 816:2007 (Department of Standards Malaysia, 2007) and PORAM has set a minimum standard of IV for RBD palm olein of 56. Alireza et al. (2010) reported that RBD palm olein bought in supermarket in Selangor, Malaysia has IV of 57.27 (packaging type was not mentioned) which was within PORAM specification. Two out of 25 cooking oils in plastic pouch had IV lower than the minimum requirement. However, their IVs were very close to 56 with IVs of 55.50 and 55.98. Notwithstanding this, sample $\mathrm{H}$ (plastic pouch) has the highest IV among all cooking oils in plastic pouches and PET bottles, with IV of 63.44. Samples V, W, R and S (PET bottle) had IV more than 60. An IV of more than 60 is considered as superolein, i.e., palm olein which has gone through double fractionation. However, Malaysian Standard for Palm Olein - MS 816:2007 (Department of Standards Malaysia, 2007) indicates that the maximum observed range for palm olein is 59.1 in which IV of more than 59.1 maybe considered as superolein. By looking only at the average IV, the 2-sample t-test concluded that cooking oils in both plastic pouch and PET bottle was significantly different $(\mathrm{p}<0.05)$ (Table $5 b)$. The commercial palm cooking oil in PET bottle has higher average IV (better) than in plastic pouch.

\section{Cloud Point}

The point when oil begins to become cloudy and no longer resist crystallisation is referred to as cloud point. As a common physical parameter, cloud point is always used to obtain rapid assessment of the oil's cold stability (Hasmadi et al., 2002). Cloud point is associated to unsaturation of oil. Lower cloud point reveals that the oil is less saturated (Zaliha et al., 2003). The range of cloud point of cooking oils
TABLE 5A. IODINE VALUE (BY CALCULATION) OF COMMERCIAL PALM-BASED COOKING OIL IN PLASTIC
POUCH AND POLYETHYLENE TEREPHTHALATE (PET) BOTTLE PACKAGING IN MALAYSIA

\begin{tabular}{lc}
\hline Sample & $\begin{array}{c}\text { Calculated iodine } \\
\text { (by calculation) }\end{array}$ \\
\hline A (PP) & $56.77 \pm 0.5^{\mathrm{hi}}$ \\
B (PP) & $56.87 \pm 0.18^{\mathrm{hi}}$ \\
C (PP) & $57.12 \pm 0.02^{\mathrm{h}}$ \\
D (PP) & $58.38 \pm 0.05^{\mathrm{g}}$ \\
E (PP) & $56.85 \pm 0.01^{\mathrm{hi}}$ \\
F (PP) & $56.88 \pm 0.02^{\mathrm{hi}}$ \\
G (PP) & $56.99 \pm 0.15^{\mathrm{h}}$ \\
H (PP) & $63.44 \pm 0.10^{\mathrm{h}}$ \\
I (PP) & $57.42 \pm 0.05^{\mathrm{cde}}$ \\
J (PP) & $58.90 \pm 0.12^{\mathrm{fg}}$ \\
K (PP) & $57.25 \pm 0.00^{\mathrm{h}}$ \\
L (PP) & $55.50 \pm 0.00^{\mathrm{j}}$ \\
M (PP) & $57.12 \pm 0.70^{\mathrm{h}}$ \\
N (PP) & $56.92 \pm 0.50^{\mathrm{h}}$ \\
O (PP) & $55.98 \pm 0.00^{\mathrm{ij}}$ \\
P (PP) & $59.33 \pm 0.20^{\mathrm{g}}$ \\
Q (PB) & $59.91 \pm 0.85^{\mathrm{de}}$ \\
R (PB) & $59.60 \pm 0.06^{\mathrm{def}}$ \\
S (PB) & $60.91 \pm 0.59^{\mathrm{bc}}$ \\
T (PB) & $60.48 \pm 0.11^{\mathrm{cd}}$ \\
U (PB) & $59.99 \pm 0.20^{\mathrm{efg}}$ \\
V (PB) & $59.83 \pm 0.00^{\mathrm{de}}$ \\
W (PB) & $62.89 \pm 0.27^{\mathrm{a}}$ \\
\hline & $51.81 \pm 0.00^{\mathrm{b}}$ \\
\hline
\end{tabular}

Note: Sample A - P - cooking oils in plastic pouch (PP).

Sample Q-Y - cooking oils in polyethylene terephthalate (PET) bottle (PB).

Means within each row bearing different superscript letters are significantly different $(\mathrm{p}<0.05)$.

TABLE 5B. 2-SAMPLE T-TEST OF IODINE VALUE (BY CALCULATION) OF COMMERCIAL PALM-BASED COOKING OIL IN PLASTIC POUCH AND POLYETHYLENE TEREPHTHALATE (PET) BOTTLE PACKAGING IN MALAYSIA

\begin{tabular}{ccc}
\hline \multicolumn{2}{c}{ Mean of IV } & p-value \\
\cline { 1 - 2 } Plastic pouch & PET bottle & \\
\hline $57.64 \pm 1.85$ & $60.26 \pm 1.38$ & 0.00 \\
\hline
\end{tabular}

Note: p-value $<0.05$ indicates that there is significant different between cooking oil in plastic pouch and PET bottle. 
in both plastic pouch and PET bottle were between $4.00^{\circ} \mathrm{C}-9.17^{\circ} \mathrm{C}$ and $4.33^{\circ} \mathrm{C}-8.00^{\circ} \mathrm{C}$, respectively (Table $6 a)$. Therefore, based on ANOVA, cloud point of all cooking oils in Malaysia (plastic pouch and PET bottle) was significantly different $(p<0.05)$ with overall range of between $4.33^{\circ} \mathrm{C}-9.17^{\circ} \mathrm{C}$. According to Teoh et al. (2005), cloud point of palm olein (from refineries in Selangor, Malaysia) is about $11.5^{\circ} \mathrm{C}$ while superolein is $5.0^{\circ} \mathrm{C}$. Palm olein has higher cloud point than soft oil (i.e. canola oil) as palm olein has higher content of saturated fatty acid which makes palm olein more stable for deep frying. It was also disclosed by Hasmadi et al. (2002) that cloud points of palm olein through RBD palm oil's fractionation at $15^{\circ} \mathrm{C}, 18^{\circ} \mathrm{C}$ and $21^{\circ} \mathrm{C}$, gave value of between $7.8^{\circ} \mathrm{C}-8.9^{\circ} \mathrm{C}$. Notwithstanding this, a study by Chnadhapuram and Sunkireddy (2012) showed a cloud point of $13^{\circ} \mathrm{C}$ for palm olein (from a study

TABLE 6A. 2-SAMPLE T-TEST OF CLOUD POINT OF COMMERCIAL PALM-BASED COOKING OIL IN PLASTIC POUCH AND POLYETHYLENE TEREPHTHALATE (PET) BOTTLE PACKAGING IN MALAYSIA

\begin{tabular}{cc}
\hline Sample & Cloud point $\left({ }^{\circ} \mathrm{C}\right)$ \\
\hline A (PP) & $9.00 \pm 0.00^{\mathrm{a}}$ \\
B (PP) & $8.00 \pm 0.00^{\mathrm{b}}$ \\
C (PP) & $8.83 \pm 0.29^{\mathrm{a}}$ \\
D (PP) & $6.00 \pm 0.00^{\mathrm{d}}$ \\
E (PP) & $8.00 \pm 0.00^{\mathrm{b}}$ \\
F (PP) & $8.00 \pm 0.00^{\mathrm{b}}$ \\
G (PP) & $9.00 \pm 0.00^{\mathrm{a}}$ \\
H (PP) & $4.00 \pm 0.00^{\mathrm{b}}$ \\
I (PP) & $8.00 \pm 0.00^{\mathrm{b}}$ \\
J (PP) & $8.00 \pm 0.00^{\mathrm{b}}$ \\
K (PP) & $8.00 \pm 0.00^{\mathrm{b}}$ \\
L (PP) & $9.17 \pm 0.29^{\mathrm{a}}$ \\
M (PP) & $8.83 \pm 0.29^{\mathrm{a}}$ \\
N (PP) & $7.67 \pm 0.29^{\mathrm{b}}$ \\
O (PP) & $8.83 \pm 0.29^{\mathrm{a}}$ \\
P (PP) & $8.67 \pm 0.29^{\mathrm{a}}$ \\
Q (PB) & $6.67 \pm 0.29^{\mathrm{c}}$ \\
R (PB) & $4.33 \pm 0.29^{\mathrm{g}}$ \\
S (PB) & $5.00 \pm 0.00^{\mathrm{ef}}$ \\
T (PB) & $8.00 \pm 0.00^{\mathrm{b}}$ \\
U (PB) & $6.83 \pm 0.29^{\mathrm{c}}$ \\
V (PB) & $5.00 \pm 0.00^{\mathrm{ef}}$ \\
W (PB) & $4.50 \pm 0.00^{\mathrm{fg}}$ \\
X (PB) & $6.67 \pm 0.29^{\mathrm{c}}$ \\
Y (PB) & $5.33 \pm 0.29^{\mathrm{e}}$ \\
\hline & \\
\hline
\end{tabular}

Note: Sample A-P - cooking oils in plastic pouch (PP). Sample Q-Y - cooking oils in polyethylene terephthalate (PET) bottle (PB).

Means within each row bearing different superscript letters are significantly different $(\mathrm{p}<0.05)$. in Karnataka India). This showed that cloud point of the commercial cooking oils in Malaysia were below than that reported by Teoh et al. (2005) and Chnadhapuram and Sunkireddy (2012) and most of them were in the range reported by Hasmadi et al. (2002). Samples H and L (plastic pouch) have the lowest and highest cloud point accordingly. The 2-sample t-test provided $\mathrm{p}<0.05$ (significantly different) of average cloud point for both cooking oil in plastic pouch and PET bottle (Table 6b). The test also showed that average cloud point in PET bottle was lower (better) than in plastic pouch. There was significant correlation of cloud point with FAC and TAG. Based on Pearson correlation, cloud point has negative correlation with FAC (monounsaturated and polyunsaturated) and TAG (UUU and SUU) while showing positive correlation with FAC (total saturated) and TAG (SUS and SSS).

TABLE 6B. 2-SAMPLE T-TEST OF CLOUD POINT OF COMMERCIAL PALM-BASED COOKING OIL IN PLASTIC POUCH AND POLYETHYLENE TEREPHTHALATE (PET) BOTTLE PACKAGING IN MALAYSIA

\begin{tabular}{|ccc|}
\hline \multicolumn{2}{c}{ Mean of cloud point $\left({ }^{\circ} \mathrm{C}\right)$} & p-value \\
\hline Plastic pouch & PET bottle & \\
\hline $8.00 \pm 1.30^{\mathrm{a}}$ & $5.81 \pm 1.23^{\mathrm{b}}$ & 0.00 \\
\hline
\end{tabular}

Note: $p$-value $<0.05$ indicates that there is significant different between cooking oil in plastic pouch and PET bottle.

\section{Smoke Point}

Smoke point occurs at the temperature when oil started to produce bluish smoke continuously, indicating fat breakdown to glycerol and FFA. Further breakdown of glycerol produces 2-propenal which is one of the major components for the bluish smoke (Katragadda et al., 2010). Matthäus, 2006 reported that the amount of FFA, DAG, monoacylglycerols (MAG) and volatile compounds which are the component having low molecular weight, influenced the amount of smoke produced. In this regards, better frying oil corresponds to higher smoke point and oils that have low thermal stability will have low smoke points.

Table $7 a$ shows the smoke point of cooking oils in both plastic pouch and PET bottle. The smoke point of cooking oil in plastic pouch and PET bottle were in the range of $190.00^{\circ} \mathrm{C}-218.00^{\circ} \mathrm{C}$ and $184.50^{\circ} \mathrm{C}-213.50^{\circ} \mathrm{C}$, respectively. Based on ANOVA, all cooking oils in plastic pouch and PET bottle were significantly different $(\mathrm{p}<0.05)$ with overall range of between $190.00^{\circ} \mathrm{C}-218.00^{\circ} \mathrm{C}$. However, there were cooking oils that were not significantly different from each other. Two studies reported that RBD palm 
TABLE 7A. SMOKE POINT OF COMMERCIAL PALMBASED COOKING OIL IN PLASTIC POUCH AND POLYETHYLENE TEREPHTHALATE (PET) BOTTLE PACKAGING IN MALAYSIA

\begin{tabular}{|c|c|}
\hline Sample & Smoke point $\left({ }^{\circ} \mathrm{C}\right)$ \\
\hline $\mathrm{A}(\mathrm{PP})$ & $190.00 \pm 0.00^{\mathrm{gh}}$ \\
\hline $\mathrm{B}(\mathrm{PP})$ & $214.50 \pm 0.71^{\mathrm{abc}}$ \\
\hline $\mathrm{C}(\mathrm{PP})$ & $200.00 \pm 0.00^{\text {cdefgh }}$ \\
\hline $\mathrm{D}(\mathrm{PP})$ & $210.50 \pm 0.71^{\text {abcde }}$ \\
\hline $\mathrm{E}(\mathrm{PP})$ & $208.00 \pm 0.00^{\mathrm{abcdef}}$ \\
\hline $\mathrm{F}(\mathrm{PP})$ & $202.00 \pm 0.00^{\text {bcdefg }}$ \\
\hline $\mathrm{G}(\mathrm{PP})$ & $206.00 \pm 0.00^{\text {abcdef }}$ \\
\hline $\mathrm{H}(\mathrm{PP})$ & $212.00 \pm 0.00^{\mathrm{abcd}}$ \\
\hline $\mathrm{I}(\mathrm{PP})$ & $216.00 \pm 0.00^{\mathrm{ab}}$ \\
\hline $\mathrm{J}(\mathrm{PP})$ & $218.00 \pm 0.00^{\mathrm{a}}$ \\
\hline $\mathrm{K}(\mathrm{PP})$ & $212.50 \pm 19.09^{\mathrm{abcd}}$ \\
\hline $\mathrm{L}(\mathrm{PP})$ & $200.50 \pm 0.71^{\text {bcdefg }}$ \\
\hline $\mathrm{M}(\mathrm{PP})$ & $206.50 \pm 0.71^{\text {abcdef }}$ \\
\hline $\mathrm{N}(\mathrm{PP})$ & $212.50 \pm 0.71^{\mathrm{abcd}}$ \\
\hline $\mathrm{O}(\mathrm{PP})$ & $207.00 \pm 0.00^{\text {abcdef }}$ \\
\hline $\mathrm{P}(\mathrm{PP})$ & $203.00 \pm 0.00^{\text {abcdefg }}$ \\
\hline $\mathrm{Q}(\mathrm{PB})$ & $200.50 \pm 0.71^{\text {bcdefg }}$ \\
\hline $\mathrm{R}(\mathrm{PB})$ & $197.00 \pm 0.00^{\text {defgh }}$ \\
\hline $\mathrm{S}(\mathrm{PB})$ & $213.50 \pm 0.71^{\mathrm{abc}}$ \\
\hline $\mathrm{T}(\mathrm{PB})$ & $194.00 \pm 0.00^{\mathrm{fgh}}$ \\
\hline $\mathrm{U}(\mathrm{PB})$ & $190.00 \pm 0.00^{\mathrm{gh}}$ \\
\hline $\mathrm{V}(\mathrm{PB})$ & $184.50 \pm 0.71^{\mathrm{h}}$ \\
\hline $\mathrm{W}(\mathrm{PB})$ & $195.50 \pm 0.71^{\text {efgh }}$ \\
\hline$X(P B)$ & $194.00 \pm 0.00^{\mathrm{fgh}}$ \\
\hline $\mathrm{Y}(\mathrm{PB})$ & $203.50 \pm 0.71^{\text {abcdefg }}$ \\
\hline
\end{tabular}

Note: Sample A-P - cooking oils in plastic pouch (PP).

Sample Q-Y - cooking oils in polyethylene terephthalate (PET) bottle (PB).

Means within each row bearing different superscript letters are significantly different $(\mathrm{p}<0.05)$.

olein normally has smoke point of between $212^{\circ} \mathrm{C}$ $227^{\circ} \mathrm{C}$ (Ahmad Tarmizi et al., 2016b; Ahmad Tarmizi and Ismail, 2014; 2008). Sample J (plastic pouch) has the highest smoke point followed by sample I (plastic pouch), sample B (plastic pouch) and sample $S$ (PET bottle). Samples that have smoke point below $200^{\circ} \mathrm{C}$ were samples A (plastic pouch) and $\mathrm{R}, \mathrm{T}, \mathrm{U}$, $\mathrm{V}, \mathrm{W}$ and X (PET bottle). Ahmad Tarmizi and Ismail (2007) and Ismail (2001) reported that initial smoke point of any oil for frying should be higher than $215^{\circ} \mathrm{C}$ in which this is the recommendation from the food industry. Furthermore, Katragadda et al. (2010) suggested that smoke point of below $200^{\circ} \mathrm{C}$ is not suited for deep frying. Cooking oil in PET bottle has higher samples of smoke point value below $200^{\circ} \mathrm{C}$ and therefore, might be less superior than the cooking oil in pouch packaging. $\mathrm{p}<0.05$ for average smoke point was observed for cooking oils in both plastic pouch and PET bottle through the 2-sample t-test (Table $7 b$ ). This showed that average cooking oils in both packagings were significantly different with cooking oil in plastic pouch, having higher (better) smoke point. Based on Pearson correlation, there were significant correlation between smoke point, SSS TAG and polyunsaturated FAC. Smoke point showed negative and positive correlation with SSS TAG and polyunsaturated FAC, respectively.

TABLE 7B. 2-SAMPLE T-TEST OF SMOKE POINT OF COMMERCIAL PALM-BASED COOKING OIL IN PLASTIC POUCH AND POLYETHYLENE TEREPHTHALATE (PET) BOTTLE PACKAGING IN MALAYSIA

\begin{tabular}{ccc}
\hline \multicolumn{2}{c}{ Mean of smoke point $\left({ }^{\circ} \mathrm{C}\right)$} & \multirow{2}{*}{ p-value } \\
\cline { 1 - 2 } Plastic pouch & PET bottle & \\
\hline $207.44 \pm 7.87^{\mathrm{a}}$ & $196.94 \pm 8.07^{\mathrm{b}}$ & 0.00 \\
\hline
\end{tabular}

Note: $p$-value $<0.05$ indicates that there is significant different between cooking oil in plastic pouch and PET bottle.

\section{Colour}

Colour is a common parameter to determine the oil quality. It has been broadly used as a guide of the quality of the cooking oil (Maskan, 2003). The oil darkening occurs due to the formations of the ketones, hydroperoxides, conjugated dienoic acids and hydroxides (Farhoosh et al., 2009). However, the darkening of the oil is not merely associated with the oxidative degradation. The colour of the palm-based oils is naturally darker than the other vegetable oils (Che Man and Tan, 1999). Table $8 a$ shows that the colour range of cooking oil in plastic pouch were 2.05-3.20 Red, 10.20-30.50 Yellow, 0.00-0.10 Neutral and 0.01 Blue while for PET bottle were 2.10-4.00 Red, 10.00-40.00 Yellow and 0.00-0.15 Neutral. Based on ANOVA, the colour of all cooking oils (plastic pouch and PET bottle) was significantly different $(\mathrm{p}<0.05)$ with overall range of 2.05-4.0 Red, 10.0-40.0 Yellow, 0.0-0.20 Neutral and 0.01 Blue. However, there were cooking oils that were not significantly different from each other. The maximum colour of RBD palm olein set by the Malaysian Standard for Cooking Oil - MS 682:2004 (Department of Standards Malaysia, 2004) is 4.0 Red and 40.00 Yellow for unblended oil and 6.0 Red and 60.0 Yellow for blended oil. The Malaysian Standard for Palm Olein - MS 816:2007 (Department of Standard Malaysia, 2007) and PORAM on the other hand, has set maximum colour value of 3.0 Red. All of the cooking oils met the specification by Malaysian Standard of Cooking Oil - MS 682:2004 (Department of Standards Malaysia, 2004). Eleven samples out of 25 cooking oil samples had red value beyond the limit set by Malaysian Standard for Palm 
Olein - MS 816:2007 (Department of Standards Malaysia, 2007) and PORAM in which five samples were in plastic pouch while six samples were in PET bottle. Ahmad Tarmizi et al. (2016b) also confirmed that the colour (red) values of palm oil, single- and double- fractionated palm olein and palm-based shortening were in the range of 2.1-3.1 Red. Sample V (PET bottle) had the highest red while sample I (plastic pouch) had the lowest red value. Karimah (2005) reported that the colour of standard palm olein was 2.6 Red compared to special quality palm olein which was 1.2 Red. Results in Table $8 a$ shows that 13 out of 25 samples had red value below 3.0 Red. The highest and lowest yellow values were detected in PET bottle which were sample U (40.0

TABLE 8A. COLOUR OF COMMERCIAL PALM-BASED COOKING OIL IN PLASTIC POUCH AND POLYETHYLENE TEREPHTHALATE (PET) BOTTLE PACKAGING IN MALAYSIA

\begin{tabular}{|c|c|c|c|c|}
\hline Sample & Red & Yellow & Neutral & Blue \\
\hline $\mathrm{A}(\mathrm{PP})$ & $3.20 \pm 0.00^{\mathrm{bc}}$ & $20.10 \pm 0.00^{\mathrm{bcd}}$ & $0.10 \pm 0.00^{\mathrm{abc}}$ & ND \\
\hline $\mathrm{B}(\mathrm{PP})$ & $2.25 \pm 0.07^{\text {gh }}$ & $10.20 \pm 0.28^{\mathrm{d}}$ & $0.05 \pm 0.07^{\mathrm{bc}}$ & ND \\
\hline $\mathrm{C}(\mathrm{PP})$ & $3.10 \pm 0.00^{\mathrm{bc}}$ & $20.00 \pm 0.00^{\mathrm{bcd}}$ & $0.00 \pm 0.00^{c}$ & ND \\
\hline $\mathrm{D}(\mathrm{PP})$ & $2.45 \pm 0.07^{\text {efgh }}$ & $12.00 \pm 0.00^{\mathrm{d}}$ & $0.10 \pm 0.00^{\mathrm{abc}}$ & ND \\
\hline $\mathrm{E}(\mathrm{PP})$ & $3.10 \pm 0.00^{\mathrm{bc}}$ & $31.00 \pm 1.41^{\mathrm{ab}}$ & $0.20 \pm 0.00^{\mathrm{a}}$ & ND \\
\hline $\mathrm{F}(\mathrm{PP})$ & $2.85 \pm 0.21^{\text {cdef }}$ & $21.00 \pm 1.41^{\mathrm{bcd}}$ & $0.00 \pm 0.00^{c}$ & ND \\
\hline G (PP) & $3.00 \pm 0.00^{\mathrm{bcd}}$ & $15.50 \pm 6.36^{\mathrm{bcd}}$ & $0.00 \pm 0.00^{c}$ & $01 \pm 0.00$ \\
\hline $\mathrm{H}(\mathrm{PP})$ & $2.25 \pm 0.07^{\mathrm{gh}}$ & $30.50 \pm 13.44^{\mathrm{abc}}$ & $0.15 \pm 0.07^{\mathrm{ab}}$ & IND \\
\hline $\mathrm{I}(\mathrm{PP})$ & $2.05 \pm 0.07^{\mathrm{h}}$ & $20.50 \pm 4.95^{\mathrm{bcd}}$ & $0.00 \pm 0.00^{c}$ & \\
\hline $\mathrm{J}(\mathrm{PP})$ & $2.80 \pm 0.42^{\text {cdef }}$ & $30.50 \pm 13.44^{\mathrm{abc}}$ & $0.10 \pm 0.00^{\mathrm{abc}}$ & ND \\
\hline $\mathrm{K}(\mathrm{PP})$ & $2.90 \pm 0.00^{\text {cde }}$ & $30.00 \pm 0.00^{\text {abc }}$ & $0.15 \pm 0.07^{\mathrm{ab}}$ & ND \\
\hline $\mathrm{L}(\mathrm{PP})$ & $2.40 \pm 0.14^{\mathrm{fgh}}$ & $20.10 \pm 0.00^{\text {bcd }}$ & $0.00 \pm 0.00^{c}$ & ND \\
\hline $\mathrm{M}(\mathrm{PP})$ & $3.15 \pm 0.07^{\mathrm{bc}}$ & $30.00 \pm 0.00^{\mathrm{abc}}$ & $0.10 \pm 0.00^{\mathrm{abc}}$ & ND \\
\hline $\mathrm{N}(\mathrm{PP})$ & $2.15 \pm 0.07^{\mathrm{gh}}$ & $20.00 \pm 0.00^{\mathrm{bcd}}$ & $0.10 \pm 0.00^{\mathrm{abc}}$ & ND \\
\hline $\mathrm{O}(\mathrm{PP})$ & $2.40 \pm 0.14^{\mathrm{fgh}}$ & $13.50 \pm 0.70^{\text {cd }}$ & $0.00 \pm 0.00^{c}$ & ND \\
\hline $\mathrm{P}(\mathrm{PP})$ & $3.10 \pm 0.00 b^{c}$ & $30.00 \pm 0.00^{\mathrm{abc}}$ & $0.00 \pm 0.00^{c}$ & ND \\
\hline $\mathrm{Q}(\mathrm{PB})$ & $3.10 \pm 0.00^{\mathrm{bc}}$ & $26.50 \pm 4.95^{\mathrm{abcd}}$ & $0.10 \pm 0.00^{\mathrm{abc}}$ & ND \\
\hline $\mathrm{R}(\mathrm{PB})$ & $3.40 \pm 0.14^{b}$ & $30.00 \pm 0.00^{\mathrm{abc}}$ & $0.05 \pm 0.07^{\mathrm{bc}}$ & ND \\
\hline $\mathrm{S}(\mathrm{PB})$ & $2.10 \pm 0.00^{\mathrm{h}}$ & $10.10 \pm 0.00^{\mathrm{d}}$ & $0.10 \pm 0.00^{\mathrm{abc}}$ & ND \\
\hline $\mathrm{T}(\mathrm{PB})$ & $3.10 \pm 0.00^{\mathrm{bc}}$ & $23.00 \pm 1.41^{\mathrm{abcd}}$ & $0.10 \pm 0.00^{\mathrm{abc}}$ & ND \\
\hline $\mathrm{U}(\mathrm{PB})$ & $3.15 \pm 0.07^{\mathrm{bc}}$ & $40.00 \pm 0.00^{\mathrm{a}}$ & $0.10 \pm 0.00^{\mathrm{abc}}$ & ND \\
\hline$V(P B)$ & $4.00 \pm 0.00^{\mathrm{a}}$ & $20.60 \pm 0.57^{\mathrm{bcd}}$ & $0.00 \pm 0.00^{c}$ & ND \\
\hline $\mathrm{W}(\mathrm{PB})$ & $2.60 \pm 0.14^{\operatorname{defg}}$ & $10.00 \pm 0.00^{\mathrm{d}}$ & $0.00 \pm 0.00^{c}$ & ND \\
\hline $\mathrm{X}(\mathrm{PB})$ & $3.00 \pm 0.14 \mathrm{bcd}$ & $30.15 \pm 0.21^{\mathrm{abc}}$ & $0.05 \pm 0.07^{\mathrm{bc}}$ & ND \\
\hline $\mathrm{Y}(\mathrm{PB})$ & $2.30 \pm 0.00 \mathrm{gh}$ & $20.05 \pm 0.07^{\mathrm{bcd}}$ & $0.15 \pm 0.07^{\mathrm{ab}}$ & ND \\
\hline
\end{tabular}

Note: Sample A-P - cooking oils in plastic pouch (PP). Sample Q-Y - cooking oils in polyethylene terephthalate (PET) bottle (PB).

Means within each row bearing different superscript letters are significantly different $(\mathrm{p}<0.05)$.
Yellow) and W (10.0 Yellow), respectively. Karimah (2005) claimed that the yellow value of special quality of palm olein is 12 Yellow. The difference in the colour of palm olein is affected from the refining process. Crude palm oil is refined to obtain desirable attributes such as neutral taste, light colour and stable from oxidation (Gibon et al., 2007; Sampaio et al., 2011). The refining process includes bleaching and deodorisation/deacidification step which eliminates the colour of the crude palm oil (Silva et al., 2014). Rossi et al. (2001) added that the colour of the finished palm oil is totally depending on the temperature as compared to the treatment with adsorbent clays and synthetic silica. It can be concluded from the 2-sample t-test that the average colour (red, yellow, neutral and blue) of cooking oil in both plastic pouch and PET bottle were comparable $(\mathrm{p}>0.05)$ (Table $8 b)$.

TABLE 8B. 2-SAMPLE T-TEST OF COLOUR OF COMMERCIAL PALM-BASED COOKING OIL IN PLASTIC POUCH AND POLYETHYLENE TEREPHTHALATE (PET) BOTTLE PACKAGING IN MALAYSIA

\begin{tabular}{|c|c|c|c|}
\hline \multirow{2}{*}{ Colour } & \multicolumn{2}{|c|}{ Mean } & \multirow{2}{*}{ p-value } \\
\hline & Plastic pouch & PET bottle & \\
\hline $\mathrm{Re}$ & $2.70 \pm 0.41$ & $2.97 \pm 0.56$ & 0.08 \\
\hline Yellow & $22.18 \pm 8.05$ & $23.38 \pm 9.48$ & 0.66 \\
\hline Neutral & $0.07 \pm 0.07$ & $0.07 \pm 0.06$ & 0.72 \\
\hline
\end{tabular}

Note: $p$-value $<0.05$ indicates that there is significant different between cooking oil in plastic pouch and PET bottle.

\section{Polar Compound}

Deteriorative measurement of cooking oil is mostly conducted through polar compound analysis (Mohamed Sulieman et al., 2006) especially for inused and discarded frying oil as it is non-volatile and thus, exhibit major reactions in the oil. Dobarganes et al. (2003) described that polar compound may consist of oxidised and polymerised TAG, DAG and FFA. Thus, it also includes hydroperoxides, aldehydes, acids, alcohols, ketones and epoxides (Melton et al., 1994). Slower accumulation of polar compound in heated oil without food compared to the addition of food was reported by Chen et al. (2013).

Polar compound of cooking oils in both plastic pouch and PET bottle were $6.02 \%-8.65 \%$ and $6.38 \%-8.43 \%$, accordingly (Table 9a). Overall polar compound of the cooking oils in both packagings in Malaysia were found to be in the range of $6.02 \%$ $8.65 \%$ and they were significantly different $(\mathrm{p}<0.05)$ from one another based on ANOVA. However, there were cooking oils that were not significantly different from each other. Polar compound of fresh palm olein was reported to be $9.8 \%$ by Tabee et al. (2009). Tarmizi 
TABLE 9A. POLAR COMPOUND OF COMMERCIAL PALM-BASED COOKING OIL IN PLASTIC POUCH AND POLYETHYLENE TEREPHTHALATE (PET) BOTTLE PACKAGING IN MALAYSIA

\begin{tabular}{|c|c|}
\hline Sample & Polar compound $(\%)$ \\
\hline $\mathrm{A}(\mathrm{PP})$ & $6.98 \pm 0.11^{\text {defghij }}$ \\
\hline $\mathrm{B}(\mathrm{PP})$ & $6.54 \pm 0.06^{\text {ghijk }}$ \\
\hline $\mathrm{C}(\mathrm{PP})$ & $7.23 \pm 0.15^{\text {defgh }}$ \\
\hline $\mathrm{D}(\mathrm{PP})$ & $7.62 \pm 0.13^{\text {bcde }}$ \\
\hline $\mathrm{E}(\mathrm{PP})$ & $7.69 \pm 0.07^{\mathrm{bcd}}$ \\
\hline $\mathrm{F}(\mathrm{PP})$ & $6.15 \pm 0.21^{1 \mathrm{k}}$ \\
\hline $\mathrm{G}(\mathrm{PP})$ & $7.22 \pm 0.00^{\text {defgh }}$ \\
\hline $\mathrm{H}(\mathrm{PP})$ & $8.65 \pm 0.21^{\mathrm{a}}$ \\
\hline $\mathrm{I}(\mathrm{PP})$ & $7.43 \pm 0.19^{\text {cde }}$ \\
\hline $\mathrm{J}(\mathrm{PP})$ & $7.52 \pm 0.16^{\text {cde }}$ \\
\hline $\mathrm{K}(\mathrm{PP})$ & $6.92 \pm 0.14^{\text {defghij }}$ \\
\hline $\mathrm{L}(\mathrm{PP})$ & $6.19 \pm 0.32^{\mathrm{jk}}$ \\
\hline $\mathrm{M}(\mathrm{PP})$ & $6.49 \pm 0.04^{\text {ghijk }}$ \\
\hline $\mathrm{N}(\mathrm{PP})$ & $6.02 \pm 0.06^{\mathrm{k}}$ \\
\hline $\mathrm{O}(\mathrm{PP})$ & $6.27 \pm 0.47^{\mathrm{ijk}}$ \\
\hline $\mathrm{P}(\mathrm{PP})$ & $6.66 \pm 0.18^{\text {fghijk }}$ \\
\hline $\mathrm{Q}(\mathrm{PB})$ & $6.38 \pm 0.07^{\text {hijk }}$ \\
\hline $\mathrm{R}(\mathrm{PB})$ & $6.64 \pm 0.18^{\text {fghijk }}$ \\
\hline$S(P B)$ & $8.43 \pm 0.38^{\mathrm{ab}}$ \\
\hline $\mathrm{T}(\mathrm{PB})$ & $7.31 \pm 0.45^{\text {defg }}$ \\
\hline $\mathrm{U}(\mathrm{PB})$ & $7.30 \pm 0.18^{\text {defg }}$ \\
\hline $\mathrm{V}(\mathrm{PB})$ & $8.33 \pm 0.06^{\mathrm{abc}}$ \\
\hline $\mathrm{W}(\mathrm{PB})$ & $7.74 \pm 0.01^{\mathrm{bcd}}$ \\
\hline $\mathrm{X}(\mathrm{PB})$ & $7.09 \pm 0.16^{\text {defghi }}$ \\
\hline $\mathrm{Y}(\mathrm{PB})$ & $6.78 \pm 0.25^{\text {efghijk }}$ \\
\hline
\end{tabular}

Note: Sample A-P - cooking oils in plastic pouch (PP). Sample Q-Y - cooking oils in polyethylene terephthalate (PET) bottle (PB).

Means within each row bearing different superscript letters are significantly different $(\mathrm{p}<0.05)$.

et al. (2016b) and Berger (2005), however, reported that fresh palm-based oil has polar compound of $6 \%-8 \%$ due to the existence of DAG. Liquid oil on the other hand, has lower polar compound of between $2 \%-3 \%$. A survey by Sebastian et al. (2014) showed that fresh canola and hydrogenated vegetable oil used as frying oil in restaurant in Toronto have polar compound of $1 \%-3 \%$ only. Sample N (plastic pouch) has a lowest polar content of $6.02 \%$, while sample $\mathrm{H}$ (plastic pouch) has the highest polar content of $8.65 \%$. Three cooking oils namely sample $\mathrm{H}$ (plastic pouch), S (PET bottle) and V (PET bottle) have higher level of polar compounds of more than $8 \%$. The 2-sample t-test confirmed that the average polar compound of cooking oils both in plastic pouch and PET bottle were comparable $(\mathrm{p}>0.05)$ (Table $9 b)$.
TABLE 9B. 2-SAMPLE T-TEST OF POLAR COMPOUND OF COMMERCIAL PALM-BASED COOKING OIL IN PLASTIC POUCH AND POLYETHYLENE TEREPHTHALATE (PET) BOTTLE PACKAGING IN MALAYSIA

\begin{tabular}{ccc}
\hline \multicolumn{2}{c}{ Mean of polar compound (\%) } & \multirow{2}{*}{ p-value } \\
\cline { 1 - 2 } Plastic pouch & PET bottle & \\
\hline $6.971 \pm 0.712$ & $7.332 \pm 0.718$ & 0.096 \\
\hline
\end{tabular}

Note: $p$-value $<0.05$ indicates that there is significant different between cooking oil in plastic pouch and PET bottle.

\section{Polymer Compound}

Polymerisation is one of the stages in the oxidation reaction (Nayak et al., 2016) that normally takes place at higher frying temperatures. Polymer compounds increased as the number of frying cycle and temperature increased (Choudhary and Grover, 2013). As the polymer compounds developed, further deterioration of the oil is accelerated, escalating the viscosity of the oil, decreasing the heat transfer, foaming during frying, darkening the colour of the oil and increasing the oil absorption into the food (Choe and Min, 2007) which imparts bitterness (Maskan, 2003). Serjouie et al. (2010) reported that the polymer content of fresh RBD palm olein was $0 \%$. However, this value can escalate during frying, that makes many countries to set their own discard value for maximum level of polymer compound. The Netherlands, for example, sets $16 \%$ of polymer compound in used oil as their maximum limit (Berger, 2005). Table 10a shows that the polymer compound of cooking oils in both plastic pouch and PET bottle were in the range of $0.54 \%$ $0.83 \%$ and $0.58 \%-0.80 \%$, respectively. The polymer compound of cooking oils in both packagings was significantly different $(\mathrm{p}<0.05)$ with overall range of $0.54 \%-0.83 \%$. However, there were cooking oils that were not significantly different from each other. Notwithstanding this, their significance were very close to each other. The highest and the lowest level of polymer compounds were found in samples M $(0.54 \%)$ and B $(0.83 \%)$. Both samples were in the plastic pouch. In terms of average polymer compound, cooking oils in both plastic pouches and PET bottles were found to be comparable $(p>0.05)$ (Table 10b).

\section{Oxidative Stability}

Oxidative stability is a crucial aspect in determining the quality of cooking oil, especially those used for deep-frying. In deep-frying study, oxidative stability determination involves immersing food in oil at high temperatures $\left(165^{\circ} \mathrm{C}-190^{\circ} \mathrm{C}\right)$. At this point, the frying oil undergoes degradation such as oxidation, hydrolysis and polymerisation (Naz et al., 2004). A good quality cooking oil should have higher resistant 
TABLE 10A. POLYMER COMPOUND OF COMMERCIAL

PALM-BASED COOKING OIL IN PLASTIC POUCH AND POLYETHYLENE TEREPHTHALATE (PET) BOTTLE PACKAGING IN MALAYSIA

\begin{tabular}{|c|c|}
\hline Sample & Polymer compound $(\%)$ \\
\hline $\mathrm{A}(\mathrm{PP})$ & $0.59 \pm 0.07^{\mathrm{bc}}$ \\
\hline $\mathrm{B}(\mathrm{PP})$ & $0.54 \pm 0.03^{c}$ \\
\hline $\mathrm{C}(\mathrm{PP})$ & $0.60 \pm 0.06^{\mathrm{bc}}$ \\
\hline $\mathrm{D}(\mathrm{PP})$ & $0.76 \pm 0.03^{\mathrm{abc}}$ \\
\hline $\mathrm{E}(\mathrm{PP})$ & $0.62 \pm 0.09^{\mathrm{abc}}$ \\
\hline $\mathrm{F}(\mathrm{PP})$ & $0.66 \pm 0.03^{\mathrm{abc}}$ \\
\hline $\mathrm{G}(\mathrm{PP})$ & $0.67 \pm 0.04^{\mathrm{abc}}$ \\
\hline $\mathrm{H}(\mathrm{PP})$ & $0.71 \pm 0.09^{\mathrm{abc}}$ \\
\hline I (PP) & $0.74 \pm 0.08^{\mathrm{abc}}$ \\
\hline $\mathrm{J}(\mathrm{PP})$ & $0.59 \pm 0.03^{\mathrm{bc}}$ \\
\hline $\mathrm{K}(\mathrm{PP})$ & $0.69 \pm 0.06^{\mathrm{abc}}$ \\
\hline $\mathrm{L}(\mathrm{PP})$ & $0.69 \pm 0.08^{\mathrm{abc}}$ \\
\hline $\mathrm{M}(\mathrm{PP})$ & $0.83 \pm 0.04^{\mathrm{a}}$ \\
\hline $\mathrm{N}(\mathrm{PP})$ & $0.60 \pm 0.03^{\mathrm{bc}}$ \\
\hline $\mathrm{O}(\mathrm{PP})$ & $0.73 \pm 0.09^{\mathrm{abc}}$ \\
\hline $\mathrm{P}(\mathrm{PP})$ & $0.69 \pm 0.04^{\mathrm{abc}}$ \\
\hline $\mathrm{Q}(\mathrm{PB})$ & $0.63 \pm 0.04^{\mathrm{abc}}$ \\
\hline $\mathrm{R}(\mathrm{PB})$ & $0.63 \pm 0.64^{\mathrm{abc}}$ \\
\hline $\mathrm{S}(\mathrm{PB})$ & $0.80 \pm 0.01^{\mathrm{ab}}$ \\
\hline $\mathrm{T}(\mathrm{PB})$ & $0.67 \pm 0.04^{\mathrm{abc}}$ \\
\hline $\mathrm{U}(\mathrm{PB})$ & $0.71 \pm 0.04$ \\
\hline $\mathrm{V}(\mathrm{PB})$ & $0.58 \pm 0.04^{\mathrm{bc}}$ \\
\hline $\mathrm{W}(\mathrm{PB})$ & $0.71 \pm 0.07^{\mathrm{abc}}$ \\
\hline$X(P B)$ & $0.60 \pm 0.02^{\mathrm{bc}}$ \\
\hline $\mathrm{Y}(\mathrm{PB})$ & $0.71 \pm 0.06^{\mathrm{abc}}$ \\
\hline
\end{tabular}

Note: Sample A-P - cooking oils in plastic pouch (PP).

Sample Q-Y - cooking oils in polyethylene terephthalate (PET) bottle (PB).

Means bearing different superscript letters are significantly different $(\mathrm{p}<0.05)$.

\begin{tabular}{|c|c|c|}
\hline $\begin{array}{r}\text { TABLE 10B. 2-SAM } \\
\text { OF COMMERCI } \\
\text { PLASTIC POUCH A } \\
(\text { PET) BOTT }\end{array}$ & $\begin{array}{l}\text { ST OF POLY } \\
\text { A-BASED CO } \\
\text { YETHYLENE } \\
\text { CAGING IN }\end{array}$ & $\begin{array}{l}\text { OMPOUND } \\
\text { G OIL IN } \\
\text { PHTHALATI } \\
\text { YSIA }\end{array}$ \\
\hline \multicolumn{2}{|c|}{ Mean of polymer compound $(\%)$} & \multirow{2}{*}{ p-value } \\
\hline Plastic pouch & PET bottle & \\
\hline $0.667 \pm 0.086$ & $0.668 \pm 0.075$ & 0.980 \\
\hline
\end{tabular}

Note: $p$-value $<0.05$ indicates that there is significant different between cooking oil in plastic pouch and PET bottle. towards oxidation in order to increase its shelf life. Oxidative deterioration of fat resulted in the development of a pungent and offensive off-flavour. Table 11a shows that the OSI of cooking oil in both plastic pouch and PET bottle ranged between 20.94$27.90 \mathrm{hr}$ and 17.71-23.97 hr, respectively. The OSI of cooking oil in both packaging was significantly different $(p<0.05)$ with overall range of 17.71-27.90 $\mathrm{hr}$. However, there were cooking oils that were not significantly different from each other. Sample E (plastic pouch) had the highest OSI while sample $S$ (PET bottle) had the lowest OSI. Ahmad Tarmizi et al. (2016a) reported that the OSI of palm olein is $28.1 \mathrm{hr}$ while Womeni et al. (2016) stated that the OSI of palm olein is $20.1 \mathrm{hr}$. The 2-sample t-test showed that the average OSI of cooking oils in both plastic pouch and PET bottle was significantly different $(\mathrm{p}<0.05)$, with plastic pouch cooking oil has higher

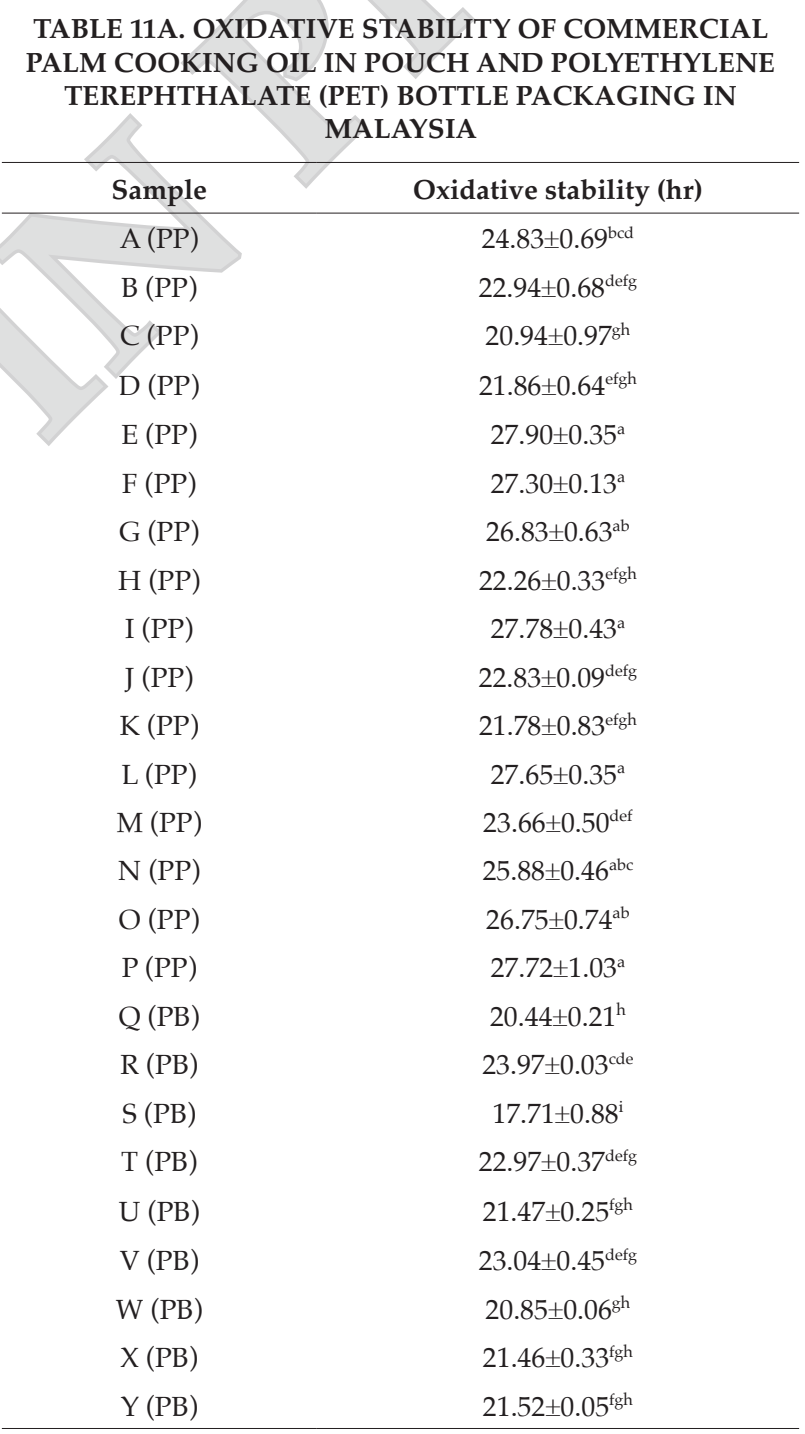

Note: Sample A-P - cooking oils in plastic pouch (PP).

Sample Q-Y - cooking oils in polyethylene terephthalate (PET) bottle (PB).

Means within each row bearing different superscript letters are significantly different $(\mathrm{p}<0.05)$. 
(better) OSI compared to PET bottle cooking oil (Table 11b). Based on Pearson correlation, there was a negative significant correlation between OSI and polyunsaturated FAC.

TABLE 11B. 2-SAMPLE T-TEST OF OXIDATIVE STABILITY OF COMMERCIAL PALM-BASED COOKING OIL IN PLASTIC POUCH AND POLYETHYLENE TEREPHTHALATE (PET) BOTTLE PACKAGING IN MALAYSIA

\begin{tabular}{ccc}
\hline \multicolumn{2}{c}{ Mean of oxidative stability (hr) } & \\
\cline { 1 - 2 } Plastic pouch & PET bottle & p-value \\
\hline $24.93 \pm 2.55$ & $21.49 \pm 1.79$ & 0.00 \\
\hline
\end{tabular}

Note: $p$-value $<0.05$ indicates that there is significant different between cooking oil in plastic pouch and PET bottle.

\section{CONCLUSION}

Most of the important quality characteristics (FFA, FAC (oleic acid), total vitamin E, colour (red, yellow, neutral and blue), polar compound and polymer compound of the commercial palm-based cooking oils sold in plastic pouches and PET bottles were statistically comparable. Therefore, consumers' perception of plastic pouch having lower quality was misleading. Notwithstanding this, from the authors perspective, better quality cooking oil was skewed towards PET bottle when other important quality characteristics of cooking oil namely FAC (palmitic acid, stearic acid and linolenic acid), TAG (UUU and SUU), IV and cloud point quality of commercial palm cooking oil in PET bottles were better than in pouches. Nevertheless, FAC and IV of plastic pouch cooking oil still met either the Malaysian Standard (MS) 682:2004, MS 816:2007 or Palm Oil Refiners' Association (PORAM) specification/guideline. In addition, the cloud point of cooking oils in plastic pouch was better than the cloud point cited in many earlier studies. TAG on the hand, does not specify in any Malaysian Standard or PORAM's specification / guideline.

\section{ACKNOWLEDGEMENT}

The authors thank the Director-General of MPOB for permission to publish this article. The authors also thank the skilled staff of Food Technology Group for their analytical assistance in ensuring the success of this study.

\section{REFERENCES}

Ahmad Tarmizi, A H and Ahmad, K (2015). Feasibility of continuous frying system to improve the quality indices of palm olein for the production of extruded product. J. Oleo Sci., 65: 1259-1266.
Ahmad Tarmizi, A H and Ismail, R (2007). Frying performance of standard of palm olein and special quality palm olein during continuous frying of potato chips. Proc. of the 2007 PIPOC International Palm Oil Congress. 26-30 August 2007. Kuala Lumpur Convention Centre, Malaysia.

Ahmad Tarmizi, A H and Ismail, R (2014). Use of pilot plant scale continuous fryer to simulate industrial production of potato chips: Thermal properties of palm olein blends under continuous frying conditions. Food Sci. Nutri., 2: 28-38.

Ahmad Tarmizi, A H and Ismail, R. (2008). Comparison of the frying stability of standard palm olein and special quality palm olein. J. Amer. Oil Chem. Soc., 85: 245-251.

Ahmad Tarmizi, A H; Ahmad, K; Mat Sahri, M and Choo, Y M (2016a). Rancimat test for measuring the oxidative stability of cooking oils upon prolonged frying: Short communication. J. Oil Palm Res. Vol. 28: 531-535.

Ahmad Tarmizi, A H; Ismail, $\mathrm{R}$ and Kuntom, A (2016b). Effect of frying on the palm oil quality attributes - A review. J. Oil Palm Res. Vol. 28: 143-153.

Ahmad Tarmizi, A H; Niranjan, $\mathrm{K}$ and Gordon, M (2013). Physico-chemical changes occurring in oil when atmospheric frying is combined with postfrying vacuum application. Food Chem., 136: 902-908.

Aladedunye, F; Kersting, H J and Matthäus, B (2014). Phenolic extract from wild rose hip with seed: composition, antioxidant capacity, and performance in canola oil. Eur. J. Lipid Sci. Technol., 116: 1025-1034.

Alireza, S; Tan, C P; Hamed, M and Che Man, Y (2010). Effect of frying process on fatty acid composition and iodine value of selected vegetable oils and their blends. Int. Food Res. J., 17: 295-302.

Arslan, N F; Şapçi, A N; Duru; F and Kara, H (2017). A study on monitoring of frying performance and oxidative stability of cottonseed and palm oil blends in comparison with original oil. Int. J. Food Prop., 20: 704-717.

Azlina, M F; Nafeeza, M I and Khalid, B A (2005). A comparison between tocopherol and tocotrienol effects on gastric parameters in rats exposed to stress. Asia Pac. J. Clin. Nutr., 14: 358-365.

Bensmira, M; Jiang, B; Nsabimana, C and Jian, T (2007). Effect of lavender and thyme incorporation in sunflower seed oil on its resistance to frying temperatures. Food Res. Int., 40: 341-346. 
Berger, K G (2005). The Use of Palm Oil in Frying. Malaysian Palm Oil Council, Selangor, Malaysia.

Bracco, U; Dieffenbacher, A and Kolarovic, L (1981). Frying performance of palm oil liquid fractions. J. Amer. Oil Chem. Soc., 58: 6-12.

Calliauw, G; Gibon, V; Greyt, W D; Plees, L; Foubert, I and Dewettinck, K (2007). Phase composition during palm olein fractionation and its effect on soft PMF and superolein quality. J. Amer. Oil. Chem. Soc., 84: 885-891.

Che Man, Y B and Tan, C P (1999). Effects of natural antioxidants on changes in refined, bleached and deodorized palm olein during deep-fat frying of potato chips. J. Amer. Oil Chem. Soc., 76: 331-339.

Chen, W-A; Chiu, C P; Cheng, W-C; Hsu, C-K and Kuo, M-I (2013). Total polar compounds and acid values of repeatedly used frying oils measured by standard and rapid method. J. Food Drug Anal., 21: 58-65.

Chnadhapuram, M and Sunkireddy, Y R (2012). Preparation of palm olein enriched with medium chain fatty acids by lipase acidolysis. Food Chem., 132: 216-221.

Choe, E and Min, D B (2007). Chemistry of deep-fat frying oils. J. Food Sci., 72: 77-86.

Choo, Y M; Yap, S C; Ooi, C K; Ma, A N; Goh, S H and Ong, A S H (1996). Recovered oil from palm-pressed fiber: A good source of natural carotenoids, vitamin E and sterols. J. Amer. Oil Chem. Soc., 73: 599-602.

Choudhary, M and Grover, K (2013). Effect of deepfat frying on physicochemical properties of rice bran oil blends. IOSR J. Nurs, Health Sci., 1: 1-10.

Department of Standards Malaysia (2007). MS 816:2007-Palm Olein-Specification (Second revision).

Department of Standards Malaysia (2004). MS 682:2004-Cooking Oils-Specification (First Revision).

Dobarganes, M C; Velasco, J and Dieffenbacher, A (2003). Determination of polar compounds, polymerized and oxidized triacylglycerols and diacylglycerols in oils and fats: Results of collaborative studies and the standardized method. Pure Appl. Chem., 72: 1563-1575.

Farhoosh, R; Kenari, R E and Poorazrang, H (2009). Frying stability of canola oil blended with palm olein, olive and corn oils. J. Amer. Oil Chem. Soc., 86: 71-76.
Firestone, D (1998). Official Methods and Recommended Practices of the AOCS, $5^{\text {th }}$ edition. American Oil Chemists' Society, Champaign, IL, USA.

Gibon, V; De Greyt, W and Kellens, M (2007). Palm oil refining. Eur. J. Lipid Sci. Tech., 109: 315-335.

Goh, S H; Hew, N F; Norhanom, A E and Yadar, M $C$ (1994). Inhibition of tumour growth promotion by various palm oil tocotrienols. Int. J. Cancer, 57: 529531.

Gunstone, F D (2008). Chapter 8. Oils and Fats in the Food Industry. First edition. Wiley-Blackwell, USA.

Ham, B; Shelton, R; Butler, B and Thionville, P (1998). Calculating the iodine value for marine oils from fatty acids profiles. J. Amer. Oil Chem. Soc., 75: 1445-1446.

Hasmadi, M; Nor Aini, S and Mamot, S (2002). The effect of different types of stirrer and fractionation temperatures during fractionation on the yield, characteristics and quality of oleins. J. Food Lipids, 9: 295-307.

Ismail, R (2001). The performance of palm olein during the industrial production of fried food. Paper presented at the $92^{\text {nd }}$ AOCS Annual Meeting and Expo, Minneapolis, USA.

Ismail, R (2005). Palm oil and palm olein frying applications. Asia Pac. J. Clin. Nutr., 14: 414-419.

ISO 5508:1990 (1990). Animal and vegetable fats and oils: Analysis of gas-liquid chromatography methyl ester of fatty acids. International Organization for Standardization, Switzerland.

IUPAC (1987).Standard Method 2.507:Determination of polar compounds in frying fats. Standard Methods for the Analysis of Oils, Fats and Derivatives, $7^{\text {th }} \mathrm{ed}$. International Union of Pure and Applied Chemistry, Blackwell, Oxford, United Kingdom.

Kamar, J P; Sarma, H D; Devasageyam, T P A; Nesaretnam, K and Basiron, Y (1997). Tocotrienols from palm olein as effective inhibitors of protein oxidation and lipid peroxidation in rat liver microsomes. Mol. Cell. Biochem., 170: 131-138.

Karimah, A (2005). Performance of special quality and standard palm olein in batch frying of fish nuggets. Palm Oil Developments, 43: 10-15.

Katragadda, R H; Fullana, A; Sidhu, S and CarbonellBarrachina, Á A (2010). Emissions of volatile aldehydes from heated cooking oil. Food Chem., 120: 59-65. 
Kostik, V; Memeti, S and Bauer, B (2013). Fatty acid composition of edible oils and fats. J. Hyg. Eng. Des., 4: 112-116.

Maskan, M (2003). Change in colour and rheological behaviour of sunflower seed oil during frying and after adsorbent treatment of used oil. Eur. Food Res. Technol., 218: 20-25.

Matthäus, B (2006). Utilization of high-olein rapeseed for deep-fat frying of French fries compared to other commonly used edible oils. Eur. J. Lipid Sci. Technol., 108: 200-211.

Matthäus, B (2007). Use of palm oil for frying in comparison with other high stability oils. Eur. J. Lipid Sci. Technol., 109: 400-409.

Matthäus, B; Haase, N U and Unbehend, G (2009). Chemical and sensory characteristics of products fried in high-oleic, low-linolenic rapeseed oil. J. Amer. Oil Chem. Soc., 86: 799-808.

Melton, S L; Jafar, S; Sykes, D and Trigiano, M K (1994). Review of stability measurements for frying oils and fried food flavor. J. Amer. Oil Chem. Soc., 71: 1301-1308.

Mohamed Sulieman, A E; El-Makhzangi, A and Ramadan, M F (2006). Antiradical performance and physicochemical characteristics of vegetable oils upon frying French fries: A preliminary comparative study. J. Food Lipids, 13: 259-276.

MPOB (2005). Methods of test for palm oil and palm oil products. MPOB, Bangi.

MPOB (2020). Malaysian oil palm industry performance 2019 and prospects for 2020. Lead paper presented at the Palm Oil Economic Review \& Outlook (R\&O) Seminar 2020 - Maximising Palm Oil Opportunity and Sustainability Amidst Challenges. 16 January 2020. Sheraton Imperial Hotel, Kuala Lumpur. p.3.

Mutalib, M S A; Khaza'ai, H and Wahle, K W J (2003). Palm tocotrienol rich fraction (TRF) is a more effective inhibitor of LDL oxidation and endothelial cell lipid peroxidation than $\alpha$-tocopherol in vitro. Food Res. Int., 36: 405-413.

Myat, M W; Abdulkarim, S M; Ghazali, H M and Roselina, K (2009). Physicochemical and characteristics of palm olein and peanut oil blend. J. Food Agric. Environ., 7: 175-181.

Nagendran, B; Unnithan, U R; Choo, Y M and Sundram, K (2000). Characteristics of red palm oil, a carotene- and vitamin E-rich refined oil for food uses. Food and Nutr. Bull., 21: 189-194.
Nallusamy, S (2006). The role of palm oil in the snack food industry. Presented at International Palm Oil Trade Fair and Seminar. 21-24 November 2006. Shangri-La Hotel, Kuala Lumpur, Malaysia.

Nawal, A-B; Shaista, A and Zubaida, A N B (2014). Characteristics and fatty acid composition of consumed cooking oil marketed locally in Riyadh city. Int. J. Biosci., 4: 227-238.

Nayak, P K; Dash, U; Rayaguru, K and Krishnan, K D (2016). Physico-chemical changes during repeated frying of cooked oil: A review. J. Food Biochem., 40: 371-390.

Naz, S; Sheikh, H; Siddiqi, R and Sayeed, S A (2004). Oxidative stability of olive, corn and soybean oil under different conditions. Food Chem., 88: 253-259.

Nesaretnam, K; Kor, H T; Ganesan, J; Chong, Y H; Sundram, K and Gapor, A (1992). The effect of vitamin E tocotrienols from palm oil on chemicallyinduced mammary carcinogenesis in female rats. Nutr. Res. 12: 63-75.

Nesaretnam, K; Stephan, R; Ray, D R and Philippa, D P (1998). Tocotrienols inhibit the growth of human breast cancer cells irrespective of estrogen receptor status. Lipids, 33: 461-569.

Ngah, W Z; Jarien, Z; San, M M; Marzuki, A; Top, G M; Shamaan, N A and Kadir, K A (1991). Effect of tocotrienols on hepatocarcinogenesis induced by 2-acetylaminofluorene in rats. Am. J. Clin. Nutr., 53: 1076S-1081S.

$\mathrm{Ng}, \mathrm{M} \mathrm{H}$; Choo, Y M; Ma, A N; Chuah, C H and Hashim, M A (2004). Separation of vitamin E (tocopherol, tocotrienol and tocomonoenol) in palm oil. Lipids, 39: 1031-1035.

Norazlina, M; Hermizi, H; Faizah, O; Nasrun, A S; Norliza, M and Ima-Nirwana, S (2010). Vitamin $\mathrm{E}$ reversed nicotine-induced toxic effects on bone biochemical markers in male rats. Arch Med. Sci., 6: 505-512.

Orthoefer, F T and List, G R (2007). Initial quality of frying oil. Deep Frying. AOCS Press. p. 33-48.

Peled, M; Gutfinger, T and Letan, A (1975). Effect of water and BHT on stability of cottonseed oil during frying. J. Sci. Food Agric., 26: 1655-1666.

Roiaini, M; Ardiannie, T and Norhayati, H (2015). Physicochemical properties of canola oil, olive oil and palm olein blends. Int. Food Res. J., 22: 1227-1233.

Rossi, M; Gianazza, M; Alamprese, C and Stanga, F (2001). The effect of bleaching and physical refining 
on color and minor components of palm oil. J. Amer. Oil Chem. Soc., 78: 1051-1055.

Sampaio, K A; Ceriani, R; Silva, S M; Taham, T and Meirelles, A J A (2011). Steam deacidification of palm oil. Food Bioprod. Process., 89: 383-390.

Sebastian, A; Ghazani, S M and Marangoni, A G (2014). Quality and safety of frying oils used in restaurants. Food Res. Int., 64: 420-423.

Sebedio, J L; Dobarganes, C; Margnes, G; Wester, I; Christie, W W; Dobson, G; Zwobada, F; Chardigmy, I M; Mariot, T H and Lahtinen, R (1996). Industrial production of crisps and prefried French fries using sunflower oils. Grasas Aceitas, 47: 5-13.

Sen, C K; Rink, C and Khanna, S (2010). Palm oilderived natural vitamin $\mathrm{E} \alpha$-tocotrienol in brain health and disease. J. Am. Coll. Nutr., 29: 314S-323S.

Serbinova, A E and Packer, L (1994). Antioxidant properties of $\alpha$-tocopherol and $\alpha$-tocotrienol. Method Enzymol., 234: 354-366.

Serjouie, A; Tan, C P; Mirhosseini, H and Che Man, Y (2010). Effects of vegetable-based oil blends on physicochemical properties of oils during deep-fat frying. Am. J. Food Technol., 5: 310-323.

Siddiqui, S; Ahsan, H; Khan, M R and Siddiqui, W A (2013). Protective effects of tocotrienols against lipid-induced nephropathy in experimental type-2 diabetic rats by modulation in TGF-beta expression. Toxicol. Appl. Pharmacol., 273:314-324.

Siddiqui, S; Khan, M R and Siddiqui, W A (2010). Comparative hypoglycemic and nephroprotective effects of tocotrienol rich fraction (TRF) from palm oil and rice bran oil against hyperglycemia induced nephropathy in type 1 diabetic rats. Chem. Biol. Interact., 188: 651-658.
Silva, S M; Sampaio, K A; Ceriani, R; Verhé, R; Stevens, C; Greyt, W D and Meirelles, A J A (2014). Effect of type of bleaching earth on the final color of refined palm oil. LWT - Food Sci. Technol., 59: 12581264.

Sundram, K and Nor, R M (2001). Analysis of tocotrienols in different sample matrixes by HPLC. Methods in Molecular Biology (Armstrong, A ed.). Vol. 186: Oxidative Stress Biomarkers and Antioxidant Protocols. Humana Press Inc., Totowa, New Jersey. p. 221-232.

Sundram, K; Sambanthamurthi, R and Tan, Y A (2003). Palm fruit chemistry and nutrition. Asia Pacific J. Clin. Nutr., 12: 355-362.

Tabee, E; Jägerstad, M and Dutta, P C (2009). Frying quality characteristics of French fries prepared in refined olive oil and palm olein. J. Amer. Oil Chem. Soc., 86: 885-893.

Teoh, C M; Nazaruddin, R; Ooi, T L; Mamot, S and Zalifah, K (2005). Strategies for decreasing the pour point and cloud point of palm oil products. Eur. J. Lipid Sci. Technol., 107: 505-512.

Womeni, H M; Djikeng, F T; Anjaneyulu, B; Karuna, M S L; Prasad, R B N and Linder, M (2016). Oxidative stabilization of RBD palm olein under forced storage conditions by old Cameroonian green tea leaves methanolic extract. NFS Journal, 3: 33-40.

Yousefi, M; Nateghi, L and Rezaee, K (2013). Investigation of physicochemical properties, fatty acids profile and sterol content in Malaysian coconut and palm oil. Ann. Biol. Res., 4: 214-219.

Zaliha, O; Chong, C L; Cheow, C S; Norizzah, A R and Kellens, M J (2003). Crystallization properties of palm oil by dry fractionation. Food Chem., 86: 245-250. 